\title{
Regulation of 15-hydroxyprostaglandin dehydrogenase expression in hepatocellular carcinoma
}

Luis Castro-Sánchez ${ }^{1}$, Noelia Agra ${ }^{1}$, Cristina Llorente Izquierdo ${ }^{1}$, Omar Motiño $^{1}$, Marta

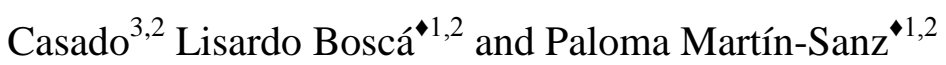

${ }^{1}$ Instituto de Investigaciones Biomédicas Alberto Sols, CSIC-UAM. Madrid. Arturo Duperier, 4, 28029 Madrid, Spain,

${ }^{2}$ Centro de Investigación Biomédica en Red de Enfermedades Hepáticas y Digestivas (CIBERehd), Córcega 180, 08036 Barcelona, Spain,

${ }^{3}$ Instituto de Biomedicina de Valencia, IBV-CSIC, Jaime Roig 11, 46010 Valencia, Spain. Short title: 15-PGDH is downregulated in HCC

\To whom correspondence should be addressed: Dr. Paloma Martín Sanz, Instituto de Investigaciones Biomédicas Alberto Sols, CSIC-UAM. Madrid. Arturo Duperier, 428029 Madrid, Spain, Tel: +0034 914972746, Fax: +0034 914973167; E-mail: pmartins@iib.uam.es or Dr. Lisardo Boscá; E-mail lbosca@iib.uam.es 


\section{ABSTRACT}

Cyclooxygenase-2 (COX-2), a rate limiting step in arachidonic acid cascade, plays a key role in the biosynthesis of prostaglandin $\mathrm{E}_{2}\left(\mathrm{PGE}_{2}\right)$ upon inflammatory stimuli, growth factors, hormones and other cellular stresses. Overproduction of $\mathrm{PGE}_{2}$ stimulates proliferation of various cancer cells, confers resistance to apoptosis and favors metastasis and angiogenesis. The steady-state level of $\mathrm{PGE}_{2}$ is maintained by interplay between the biosynthetic pathway including $\mathrm{COX}$ and $\mathrm{PGE}_{2}$ synthases and the catabolic pathways involving nicotinamide adenine dinucleotide $\left(\mathrm{NAD}^{+}\right)$-dependent 15-hydroxyprostaglandin dehydrogenase (15PGDH). 15-PGDH is a crucial enzyme responsible for the biological inactivation of $\mathrm{PGE}_{2 .}$ Adult hepatocytes fail to induce COX-2 expression regardless of the pro-inflammatory factors used. COX-2 is induced in hepatocytes after partial hepatectomy $(\mathrm{PH})$, in animal models of cirrhosis, in human hepatoma cell lines, in human HCC and after HBV and HCV infection. However, no data are available regarding 15-PGDH expression in HCC. Our results show that 15-PGDH is downregulated in human hepatoma cells with a high COX-2 expression, in chemical and genetic murine models of HCC and in human HCC biopsies. Moreover, 15PGDH expression is suppressed by EGF (epidermal growth factor) and HGF (hepatocyte growth factor) mainly involving PI3K (phosphatidylinositol-3-kinase), ERK (extracellular signal-regulated kinase) and p38MAPK (mitogen-activated protein kinase) activation. Conversely, ectopic expression of 15-PGDH induces apoptosis in hepatoma cells and decreases the growth of hepatoma cells in nude mice whereas the silencing of 15-PGDH increases the tumor formation. These data suggest a potential therapeutic application of 15PGDH in HCC. 


\section{INTRODUCTION}

Hepatocellular carcinoma (HCC) is the fifth most common cancer worldwide and has an increasing incidence in western countries (Llovet and Bruix, 2008). Although the risk factors for HCC are well characterized, the molecular pathogenesis of this tumor type is not well understood, and thus the identification of new possible targets for the development of nonconventional treatments is urgent and must be improved (Hoshida et al. , 2009, Lee et al., 2004).

Cyclooxygenase-1 (COX-1) and -2 catalyze the first step in prostanoid biosynthesis. COX-1 is constitutively expressed in many tissues, whereas $\mathrm{COX}-2$ is induced by a variety of stimuli such as growth factors, pro-inflammatory stimuli, hormones and other cellular stresses (Simmons et al. , 2004). Adult hepatocytes fail to induce COX-2 expression regardless of the pro-inflammatory factors used (Martin-Sanz et al. , 1998). However, our group and others demonstrated that partial hepatectomy $(\mathrm{PH})$ induced $\mathrm{COX}-2$ in hepatocytes and contributed to the progression of cell cycle after PH (Casado et al. , 2001, Rudnick et al. , 2001). In addition to liver regeneration after $\mathrm{PH}$, expression of $\mathrm{COX}-2$ has been observed in animal models of cirrhosis (Yamamoto et al. , 2003), in human hepatoma cell lines (Kern et al. , 2006, Mayoral et al. , 2005), in human HCC (Cusimano et al. , 2009) and after HBV and HCV infection (Cheng et al. , 2008, Nunez et al. , 2004). The second key enzyme that couples with COX-2 for the synthesis of $\mathrm{PGE}_{2}$ is $\mathrm{PGE}_{2}$ synthase (PGES). Three types of PGES participating in the synthesis of $\mathrm{PGE}_{2}$ have been described: one cytosolic (cPGES) and two membrane-associated PGES (mPGES-1 and -2). Coupling with COXs, these two types of PGES display different functions. cPGES is a cytosolic protein identical to the Hsp90-associated protein 23, which is a cofactor for the molecular chaperone function of Hsp90. It is constitutively expressed in many cell types and is predominantly coupled with COX-1. mPGES-1 is an inducible enzyme which belongs to the membrane-associated proteins involved in eicosanoid and glutathione 
metabolism (MAPEG) superfamily, catalyzes the synthesis of $\mathrm{PGE}_{2}$ commonly found in epithelial cells and is upregulated during inflammatory conditions (Diaz-Munoz et al. , 2012, Subbaramaiah et al. , 2004). Recently, more evidence has suggested that mPGES-1 participates in various pathophysiological states in which COX-2 is involved. Consistent with the role of mPGES-1 for PGE $_{2}$ synthesis, mPGES-1 knockdown has been shown to inhibit $\mathrm{PGE}_{2}$ production and reduce tumor proliferation and/or invasiveness of several tumor cell types including Lewis lung carcinoma cells, human prostate cancer cell lines and colorectal cancer cells, through a novel EGR1/ $\beta$-catenin signaling axis (Sasaki et al. , 2011). In tumors the steady-state level of $\mathrm{PGE}_{2}$ is maintained by the biosynthetic pathway including both types of PGES, mPGES and cPGES and the catabolic pathways involving 15-PGDH. 15PGDH is a crucial enzyme responsible for the biological inactivation of $\mathrm{PGE}_{2}$ which induces cell proliferation, migration, angiogenesis and tumor metastasis (Na et al. , 2011). 15-PGDH metabolizes $\mathrm{PGE}_{2}$ by oxidizing the $15(\mathrm{~S})$-hydroxyl group into a keto group producing 15 -keto $\mathrm{PGE}_{2}$ (Tai et al. , 2006). Genetic deletion of 15-PGDH leads to increased tissue levels of $\mathrm{PGE}_{2}$. Although previous studies on the distribution and activity of 15-PGDH have focused primarily in parturition and uterine biology, recent data suggest that 15-PGDH plays an important role in carcinogenesis. 15-PGDH is a tumor suppressor in gastrointestinal, colorectal, breast and lung cancers (Ding et al. , 2005, Wolf et al. , 2006, Yan et al. , 2004). However, a high expression of 15-PGDH as well as COX-2 was observed in a malignant ovarian tissue and the expression of 15-PGDH was induced by some cytokines, by the tumor promoter phorbol 12-myristate 13 acetate (PMA) and by sex hormone, suggesting a possible involvement of this enzyme in the carcinogenic process as well (Tong and Tai, 2004).

Keeping in mind these data we evaluated the potential role of 15-PGDH in hepatoma cells. Our results show that 15-PGDH is downregulated in those human hepatoma cells with a high COX-2 expression, in chemical and genetic murine models of $\mathrm{HCC}$ and in human liver HCC 
biopsies. Moreover, 15-PGDH expression is regulated by EGF and HGF mainly through PI3K, ERK and p38 MAPK and over expression of 15-PGDH induces apoptosis in hepatoma cells.

\section{MATERIALS AND METHODS}

2.1. Patients: Eighteen individual tumoral and paired non-tumoral frozen $\mathrm{HCC}$ tumors were obtained from de Spanish Tumor Bank Network of the Centro Nacional de Investigaciones Oncológicas (CNIO). Institutional review board approval (No PI. CEI PI 20_2011) was obtained for these studies and all participants provided written informed consent. All tissues were evaluated by pathologists by means of hematoxylin/eosin staining. Tissues were snapfrozen in liquid nitrogen and total RNA was isolated as described in supplementary.

2.1.1. Immunohistochemical staining: $2-4 \mu \mathrm{m}$ thick paraffin-embedded tissue microarrays (TMAs) and complete sections were cut onto Dako slices (DAKO, Glostrup, Denmark) by Immunohistochemistry Unit of CNIO, and subsequently dewaxed, rehydrated and subjected to antigen retrieval by heating in PTLink with $50 \mathrm{mM}$ Tris/EDTA, $\mathrm{pH}$ 9.0. The slides were cooled and treated with peroxidase-blocking solution (DAKO) for $5 \mathrm{~min}$. Sections were immunostained with a 15-PGDH antibody (NeoMarkers, Suffolk, UK), in a Autostainer Plus (DAKO). In parallel, each tissue section was also incubated with a goat anti-rabbit antiserum (negative control). Microphotographs were taken with an EnVision FLEX system. The images were processed with Image J software. Integrated density was calculated for each image after background subtraction. The background corresponds to the immunoreactivity in the positive control tissue (normal liver).

2.1.2. Animal models: Mice (25-30g body weight) on a C57BL6JxDBA background were used in this study. The animals were housed on a $12 \mathrm{~h}$ light/12 h dark cycle in an air conditioned room at $25^{\circ} \mathrm{C}$ with food and water available ad libitum. To induce $\mathrm{HCC}$, diethylnitrosamine (DEN) (25 mg/Kg body weight, Sigma) was injected intraperitoneally 
(i.p.) into 14 day old mice. Mice were sacrificed at 10 and $40 \mathrm{wk}$ after DEN treatment, their body and liver weights were recorded and their livers removed and separated into individual lobes. Mice were characterized as described by Llorente Izquierdo et al. (Llorente Izquierdo et al. , 2011). Liver tissues were snap frozen in liquid nitrogen and stored at $-80^{\circ} \mathrm{C}$. Moreover, a genetic model of HCC was used. Alb-c-myc (c-myc) and MT-TGF- $\alpha$ (TGF- $\alpha$ ) singletransgenic mice were kindly obtained from Dr. S.S. Thorgeirsson (National Cancer Institute, Bethesda, Maryland). Generation of c-myc/TGF- $\alpha$ double transgenic mice was achieved by crossing homozygous B6CBA c-myc and CD-1 TGF- $\alpha$ mouse as described previously (Santoni-Rugiu et al. , 1998). Expression of TGF- $\alpha$ driven by metallothionein I promoter was maintained by giving mice $50 \mathrm{mM} \mathrm{ZnCl} 2$ in drinking water starting at weaning. All the experiments were performed in accordance with the animal care guidelines of the European Union (2010/63/EU), and approved by the Bioethical Committee from Consejo Superior de Investigaciones Científicas (reference project SAF2010/16037). Male were sacrificed after 5 and 9 months of age and their body and liver weights were recorded before the liver tissues were used for analysis. Wt mice were sacrificed at 9 months-old. Externally visible tumors $(\geq$ $0.5 \mathrm{~mm}$ ) were analyzed using the Metamorph software (Universal Imaging Corp, West Chester, PA, USA). Liver tissues were snap frozen in liquid nitrogen and stored at $-80^{\circ} \mathrm{C}$ or fixed in $10 \%$ buffered formalin. Plasma was obtained from cardiac puncture or from the aorta. 2.1.3. Cell culture: The human liver cell lines WRL68, Chang liver (CHL) and the hepatoma cell lines HepG2 and Hep3B were purchased from the American Type Culture Collection (ATCC, Manassas, VA, USA). HuH-6 and HuH-7 cell lines were kindly provided by Dr. Perret (Institut Cochin, CNRS UMR8104, University Paris-Descartes, Paris, France) (de La Coste et al. , 1998) and Dr. Kern (Department of General Pathology, University Hospital Heidelberg, Heidelberg, Germany) (Kern et al. , 2002), respectively. Human cell lines authentication and intra-species cell line cross-contamination were analyzed using 
Promega's StemElite ${ }^{\mathrm{TM}}$ ID System for the following STRs (short tandem repeats): D21S11, TH01, TPOX, vWA, CSF1PO, D16S539, D7S820, D13S317 and D5S818; Amelogenin was also analyzed for gender identification together with an specific marker for mouse DNA. STRs were analyzed with the Applied Biosystems® 3130xl Genetic Analyzer in the Genomics Core Facility of the Instituto de Investigaciones Biomédicas Alberto Sols CSICUAM. Cells were expanded twice, and stored in liquid $\mathrm{N}_{2}$. Expansions from these clones were used up to 6 months in culture. Cells were grown on DMEM supplemented with $10 \%$ FBS and antibiotics (50 $\mu \mathrm{g}$ each of penicillin, streptomycin and gentamicin per ml). The cells were treated with EGF, HFG, $5 \mu \mathrm{M} \mathrm{PGE}_{2}$ and the following pharmacological inhibitors: 1

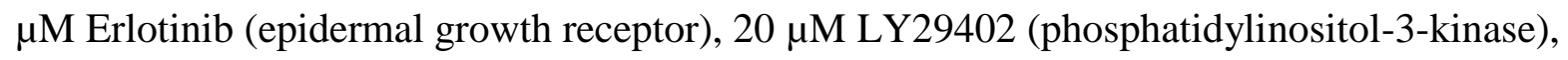

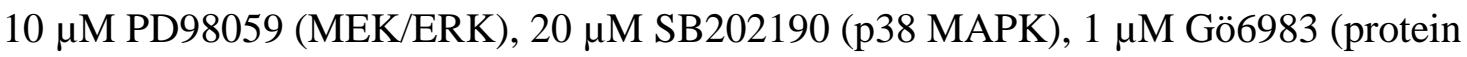
kinase C) and $5 \mu \mathrm{M}$ DFU (5,5-dimethyl-3(3-fluorophenyl)-4-(4-methylsulfonyl) phenyl2(5H)-furanone) (COX-2) (MSD; Rhaway, NJ, USA). Timing and concentration curves were performed previously to determine the optimal concentrations of EGF.

2.1.4. Analysis of tumorigenicity in nude mice: Female athymic nu/nu mice (6 weeks old) were obtained from Charles River Laboratories (Wilmington, MA). The animals were kept under pathogen-free conditions and were given an autoclaved standard diet and water ad libitum. HepG2 cells were transfected with pcDNA3/15-PGDH $(3 \mu \mathrm{g})$ or control vector $(3 \mu \mathrm{g})$ and with $30 \mathrm{nM}$ siPGDH or 30nM scRNA using lipofectamine 2000 (Invitrogen, USA) according to the manufacturer's instructions. At $48 \mathrm{~h}$ after transfection, $10 \times 10^{6}$ viable cells suspended in PBS were injected subcutaneously into both flanks of the $n u / n u$ mice ( 8 mice per group). Tumor growth was measured every 2 or 3 days. At 21 days after injection, mice were killed and tumors were weighed after necropsy. Tumor volume (V) was monitored by measuring the length (L) and width (W) with calipers and calculated with the formula (L x $\left.\mathrm{W}^{2}\right) \times 0.5$. 
2.1.5. Data analysis: Data are expressed as mean \pm S.D. (n ranged from three to five independent experiments or 8-12 mice per group). Statistical significance was estimated by one way analysis of variance (ANOVA) coupled Dunnett's Multiple Comparison Test for unpaired observations. Spearman $r$ test for nonparametric correlations and Mann-Whitney $U$ test was used for ordinal variables using the statistical software GraphPad Prism 5. A P value $<0.05$ was considered significant.

\section{RESULTS}

\subsection{5-PGDH and COX-2 expression correlate inversely in hepatoma cell lines. COX-2,} mPGES-1 and 15-PGDH protein and mRNA levels were analyzed in four hepatoma (HCC) cell lines (HepG2, Hep3B, HuH-7, HuH-6), in a cell line derived from human liver embryo (WRL68) and in a cell line derived from normal liver (CHL), using human hepatocytes (HH) as control (Fig. 1A-D). Each cell line expresses different levels of COX-2, mPGES-1 and 15PGDH mRNA and protein. WRL68 exhibited the highest COX-2 and mPGES-1 expression levels followed for Hep3B and HuH-7 liver carcinomas, whereas CHL, HepG2 and HuH-6 showed low levels of COX-2 and mPGES-1 (Fig. 1A-B-C). Interestingly, the COX-2 positive-expression cell lines WRL68, Hep3B and HuH-7 displayed the lowest 15-PGDH mRNA and protein levels (Fig. 1A-D). PGE $_{2}$ levels correlate with those of the COX-2 and mPGES-1 expression, as well as the decrease of 15-PGDH levels (Fig. 1E). To determine whether exits a relationship between COX-2 and 15-PGDH expression in the HCC cell lines, the COX-2 protein/15-PGDH protein ratio was calculated showing an inverse linear correlation $\left(\mathrm{R}^{2}=0.8524, \mathrm{P}=0.0238\right)$ (Fig. $\left.1 \mathrm{~F}\right)$. 


\subsubsection{5-PGDH is downregulated by EGF in hepatic human cell line.}

Previous reports indicated that EGF and HGF challenges upregulate COX-2 and downregulate 15-PGDH expression in several carcinomas (Donnini et al. , 2011, Moore et al. , 2009, Tai et al. , 2011). Our results demonstrate that EGF increased COX-2 and mPGES-1 levels in CHL cells (Fig. 2A). In addition to this, EGF decreased 15-PGDH protein levels in a concentration-dependent manner (Fig. 2A). Moreover, treatment of CHL cells with $50 \mathrm{ng} / \mathrm{ml}$ of EGF induced a marked increase of COX-2 and mPGES-1 protein and mRNA levels that reached a maximum at $48 \mathrm{~h}$ and $6 \mathrm{~h}$, respectively (Fig. 2B-C). Furthermore, $50 \mathrm{ng} / \mathrm{ml}$ of EGF downregulated 15-PGDH protein and mRNA levels (Fig. 2B). Next, we decided to investigate the participation of HGF in the regulation of 15-PGDH expression. HGF induced COX-2 and mPGES-1 and reduced the 15-PGDH protein levels in CHL cells in a concentrationdependent manner (Fig. 2D). To substantiate further our results, we examined whether EGF participates in the regulation of 15-PGDH expression in the hepatoma cell lines HepG2 and HuH-6. EGF induced mPGES-1 and COX-2 expression in HepG2 and HuH-6 cells (Supplementary Fig. S1A-B). Additionally, EGF decreased 15-PGDH expression in a concentration-dependent manner in HepG2 and HuH-6 cell lines (Supplementary Fig. S1AB).

\subsubsection{5-PGDH decrease induced by EGF involves EGFR, PI3K, MEK/ERK-MAPK and} p38-MAPK signaling pathways in CHL cells.

CHL cells were incubated with different pharmacological inhibitors of key regulatory pathways and then challenged with EGF. Fig. 3A shows that COX-2 expression was downregulated by LY29402 and Erlotinib, suggesting that EGF-induced COX-2 expression was EGFR-dependent and involved PI3K signaling. Furthermore, 15-PGDH protein levels were upregulated by LY29402, SB202190, PD98059 and Erlotinib (Fig. 3A) suggesting that 
15-PGDH expression decreased by EGF was EGFR-dependent and involved PI3K, p38 MAPK and MEK/ERK MAPK signaling. Next, we investigate whether exogenous $\mathrm{PGE}_{2}$ participates in the 15-PGDH downregulation induced by EGF in CHL cells. As Fig. 3B shows, COX-2 protein levels were not modified by treatment with DFU and PGE 2 . In parallel 15-PGDH protein levels were not altered after COX-2 inhibition with DFU; however, $\mathrm{PGE}_{2}$ treatment increased 15-PGDH protein levels, suggesting a positive feed-back mechanism, which is interrupted by EGF (Fig. 3B). To substantiate further our results, HepG2 and CHL cells were silenced for 15-PGDH using specific siRNAs. COX-2 expression increased and the opposite was found when the cells were transfected with an expression vector for 15-PGDH. Moreover, when WRL68 cells were silenced for COX-2 using specific siRNAs, 15-PGDH expression increased. These results suggest a complex interplay between COX-2 and 15PGDH in some hepatoma cells (Supplementary Fig. S2A-C).

\subsubsection{5-PGDH expression decreases HCC cell viability and induces apoptosis.}

To further evaluate the effect of 15-PGDH in HCC cells outcome, we tested whether 15PGDH targeting, modulates cell viability or cell cycle in HCC cells. Overexpression of 15PGDH significantly decreased cell viability and promoted apoptosis in HepG2 and CHL cells (Fig. 4B, D-E and Supplementary Fig. S3B, D-F) whereas silencing of 15-PGDH with a siPGDH promoted an increase in cell viability and in a subset of cells growing in S-phase (Fig. 4B-C and Fig. S3B-C-E). Furthermore, 15-PGDH overexpression induced a decrease in $\mathrm{G}_{0} / \mathrm{G}_{1}$ and $\mathrm{G}_{2}$ phase cells (Fig. 4D and S3D-F) and an increase (219\%) in apoptosis (Fig. 4E). Apoptosis was confirmed by measurement of caspase-3 activity and the expression of some pro- and anti-apoptotic proteins. 15-PGDH overexpression induced an increase in caspase-3 activity (Fig. 4F) and in the expression of the pro-apoptotic proteins Bax and Bid (Fig. 4G), whereas the expression of anti-apoptotic proteins Bcl-2 and Bcl-xL was decreased (Fig. 4H). 
These results suggest that 15-PGDH may exert its anti-tumoral effects partially through induction of apoptosis.

\subsubsection{5-PGDH decreases the growth of hepatoma cells in vivo.}

To further analyze the effect of 15-PGDH on hepatoma cell growth in vivo, HepG2 cells were targeted to modulate 15-PGDH levels and cells were subcutaneously injected into athymic $n u / n u$ mice. The mice were followed by the observation of xenograft growth for 3 weeks. We found that 15-PGDH led to a significant reduction in the volume and weight of the tumor comparing with the mice injected with control vector. Transfection with siPGDH increased the volume and the weight of tumors vs. scRNA (Fig. 4I-J). These results agree with the in vitro data and suggest that 15-PGDH inhibits the proliferation of hepatoma cells by inducing apoptosis.

\subsubsection{5-PGDH expression in murine models of HCC.}

To determine the role of 15-PGDH in HCC development, mice were injected with DEN (25 $\mathrm{mg} / \mathrm{kg}$ ) at the 14 th postnatal day and sacrificed 10 and $40 \mathrm{wk}$ after treatment. Plasma levels of liver injury markers after DEN treatment were analyzed in supplementary Table I. There was an important increase of AST and ALT after DEN treatment compared with the untreated mice. In addition, morphometric analysis revealed important differences in the number and size of detectable tumors at 40 wk compared with 10 wk after DEN treatment (Fig. 5A). This would be coupled with the histopathological analysis of the liver biopsies indicating preneoplasic lesions (8\%) in mice at $10 \mathrm{wk}$ after DEN and HCC (100\%) in mice at 40 wk after DEN and lung metastases (25\%) (Table I). Histological analysis confirmed that all tumors at $40 \mathrm{wk}$ after DEN treatment corresponded to liver carcinomas (Fig. 5B). Tumors at 40 wk after DEN were positive for $\alpha$-fetoprotein expression (not shown). Furthermore, 15PGDH decreased at $10 \mathrm{wk}$ after DEN treatment and was negligible in the non-tumor and 
tumor tissue at 40 wk after treatment compared with normal liver (Fig. 5C). We also analyzed the profile of 15-PGDH in the double transgenic mice c-myc/TGF- $\alpha$, a genetic model of HCC. Plasma levels of liver injury markers and the incidence of pathological signs are given in supplementary Table II. The morphometric analysis revealed significant differences in the number and size of detectable tumors in c-myc/TGF- $\alpha$ mice at 9 months old compared with 5 months-old mice (Fig. 5D). Most of the c-myc/TGF- $\alpha$ double transgenic mice sacrificed at 5-months-old-age had preneoplasic foci (93.3\%) (supplementary Table II). Furthermore, histological analysis of liver biopsies showed a tendency to develop adenomas (26.7\%). After 9 months, >89\% of Tg mice displayed HCC (supplementary Table II and Fig. 5E). Interestingly, 15-PGDH protein levels were undetectable in the tumors generated by cmyc/TGF- $\alpha$ stably expression (Fig. 5F) compared with normal liver. The double transgenic was verified by c-myc and TGF- $\alpha$ protein expression (Fig. 5F).

\subsubsection{Downregulation of 15-PGDH mRNA and protein expression in HCC human}

\section{biopsies.}

To extend our studies and assess the biological relevance of these observations in cell lines and murine models, we examined the 15-PGDH expression in 11 clinical samples of HCC and normal liver in a tissue microarray for immunohistochemistry analysis. A high 15-PGDH expression in normal liver tissue was detected (Fig. 6Aa), whereas in HCC tissue biopsies 15PGDH was significantly lesser expressed (Fig. 6Ab, c and 6B). Immunoreactivity of 15PGDH protein ranged from $61 \%$ to $0.09 \%$ vs. normal liver (100\%, Fig. 6B). The average of 15-PGDH immunoreactivity measurements was statistically significant compared with normal liver (Fig. 6C). In agreement with those observations, mRNA 15-PGDH expression in 18 individual tumoral (T) and paired non-tumoral (NT) HCC human samples showed higher levels in NT tissue compared to T tissue ( $\mathrm{P}=0.0322)$ (Fig. 6D). These data support the hypothesis that 15-PGDH expression is downregulated in HCC. 


\section{DISCUSSION}

In this study we have analyzed the regulation of the expression of 15-PGDH in hepatoma cell lines, murine models of HCC and human HCC specimens. Our results show that 15-PGDH is downregulated in human hepatoma cells with a high COX-2 and mPGES-1 expression, in chemical and genetic murine models of HCC and in human HCC biopsies. Moreover, 15PGDH expression is suppressed by EGF and HGF mainly involving PI3K, ERK and p38MAPK activation, which supports cell viability. Conversely, ectopic expression of 15PGDH induces apoptosis in hepatoma cells and decreases the growth of hepatoma cells in nude mice whereas the silencing of 15-PGDH increased the tumor formation by promote increased in cell viability.

Several lines of evidence suggest that COX-2 signaling is implicated in hepatocarcinogenesis and that COX-2 inhibitors prevent HCC cell growth in vitro and in animal models (Kern et al. , 2004). Increased COX-2 expression has been found in human HCC; however, although COX-2 is elevated in the early stages of HCC, many questions remain unsolved regarding the sufficiency of COX-2 to induce/contribute to tumorigenesis (Cusimano, Fodera, 2009). Our previous work demonstrated that constitutive COX-2 expression in mice liver is not sufficient to enhance malignant transformation induced by DEN (Llorente Izquierdo, Mayoral, 2011). Regarding mPGES-1, it participates in various pathophysiological states in which COX-2 is involved. Consistent with the role of mPGES-1 for PGE 2 synthesis, mPGES-1 knockdown has been shown to reduce $\mathrm{PGE}_{2}$ levels and reduce tumor proliferation and/or invasiveness in several tumor cell types through a EGR1/ $\beta$-catenin signaling axis (Donnini, Finetti, 2011, Sasaki, Kamei, 2011). The third enzyme involved in PGE $_{2}$ homeostasis, 15-PGDH, also plays an important role in carcinogenesis. 15-PGDH is downregulated and is a tumor suppressor in gastrointestinal, breast and lung cancers (Ding, Tong, 2005, Lou et al. , 2012, Wolf, O'Kelly, 2006, Yan, Rerko, 2004). 15-PGDH catalyzes the rate 
limiting step of PG catabolism and thus represents a physiological antagonist of COX-2 (Backlund et al. , 2005). Recent emerging evidence suggests that elevated $\mathrm{PGE}_{2}$ in cancers may be the result of enhanced COX-2 mediated $\mathrm{PGE}_{2}$ synthesis, but also to reduced 15PGDH-dependent degradation. Although COX-2 expression in HCC has been extensively studied there are few data concerning mPGES-1 and 15-PGDH involvement in HCC (Cusimano, Fodera, 2009, Kern, Schoneweiss, 2004, Koga et al. , 1999, Llorente Izquierdo, Mayoral, 2011). Lim et al. reported that omega-3 polyunsaturated fatty acids inhibited $\mathrm{PGE}_{2}$ signaling through decreased of COX-2 and expression of 15-PGDH in HCC cell lines (Lim et al. , 2009). In hepatoma cell lines our data show different levels of COX-2, mPGES-1 and 15-PGDH. Interestingly, there was a direct linear correlation between COX-2 and mPGES-1 and an inverse correlation between $\mathrm{COX}-2$ and 15-PGDH. $\mathrm{PGE}_{2}$ levels paralleled COX-2 and mPGES-1 expression.

The EGF and HGF signaling pathways upregulate COX-2 expression in different cancer cell types and downregulate 15-PGDH expression in several carcinomas (Donnini, Finetti, 2011, Moore, Greenhough, 2009, Tai, Chi, 2011). Inhibition of Met expression increased 15-PGDH levels and both ERK and Akt signaling pathways were required for the COX-2 rise and 15PGDH decrease (Moore, Greenhough, 2009). Backlund et al. showed that a tyrosine kinase inhibitor, Erlotinib, increased the levels of 15-PGDH in colorectal cancer cell lines and this effect was mimicked by a MEK inhibitor (Backlund, Mann, 2005). PI3K/AKT and Ras/MAPK kinase signaling cascades which are downstream EGFR engagement, are implicated in the down-regulation of 15-PGDH induced by EGF. Suppression of ERK activity blocked the effect of EGF on 15-PGDH, whereas a MEK kinase inhibitor was capable of suppressing the expression of Slug and Zeb1 which repress the expression of 15-PGDH (Yang et al. , 2007). Moreover, Snail and Slug induced by EGF receptor signaling recruit histone deacetylases (HDACs) which bind to conserved E-box elements present in the 
promoter of 15-PGDH and repress its transcription (Mann et al. , 2006). Our results agree with these data being 15-PGDH downregulated in HCC cells by EGF and HGF through PI3K, p38 MAPK, MEK/ERK MAPK and EGFR signaling dependent pathways.

An important question in this area is to address whether 15-PGDH and COX-2 are reciprocally regulated in cancer cells. In human lung adenocarcinoma cells, A549, which exhibit the capacity of expressing both COX-2 and 15-PGDH, overexpression of COX-2 resulted in downregulation of 15-PGDH and increased expression of 15-PGDH impedes the rise of COX-2 induced by IL-1 $\beta$. The lower the endogenous level of 15-PGDH expression present in the cells is, the higher the levels of COX-2 are. Since a catalytically inactive 15PGDH mutant also impeded the expression of COX-2 induced by IL-1 $\beta$ it became clear that the inhibition of COX-2 expression was not dependent on the catalytic activity of 15-PGDH (Tai, 2011). Song et al. reported similar results in gastric cancer cells where 15-PGDH expression increased as COX-2 was repressed by COX-2-siRNA (Song et al. , 2011). In the case of liver, CHL cells were shown to have the capacity of expressing COX-2, mPGES-1 and 15-PGDH. COX-2 was rarely observed under basal conditions, whereas 15-PGDH was usually present. Expression of COX-2 induced by EGF and HGF was in parallel with a drop of 15-PGDH in CHL cells; interestingly, this EFG-induced reduction of 15-PGDH was also observed in the presence of a potent and selective COX-2 inhibitor (DFU), reinforcing the view of a minimal contribution of PGs on this cross-regulation. Indeed, exogenous $\mathrm{PGE}_{2}$ also did not influence of 15-PGDH levels. Moreover, when HepG2 and CHL cells were transfected with a siRNA to downregulate 15-PGDH, COX-2 levels increased and the contrary was found with ectopic expression of 15-PGDH. Even more, downregulation of COX-2 in WRL68 cells increased 15-PGDH expression. These results suggest an activityindependent interplay between COX-2 and 15-PGDH in hepatoma cells, perhaps in the same way as reported by Tong et al. that proposed a direct interaction of 15-PGDH with the COX-2 
3'UTR through its AU-rich sequences that destabilize its mRNA blocking translation (Tong et al. , 2006).

There are different reports concerning the role of 15-PGDH in in vivo models of colon cancer. In the APC ${ }^{\mathrm{Min}}$ mouse that spontaneously develop preinvasive adenomas that recapitulate many aspects of the human syndrome, familial adenomatous polyposis coli pathology, 15PGDH was observed in the well differentiated villi from wild type mice, whereas it was absent in adenomas from APC Min/+ mice (Backlund et al. , 2008, Mann, Backlund, 2006). Using a 15-PGDH knockout mouse model, Yan et al. showed a remarkable sensitization to colon tumor induction by azoxymethane and this deficiency in $\mathrm{APC}^{\mathrm{Min}}$ resulted in an eightfold increase in colon tumor development $v s . \mathrm{APC}^{\mathrm{Min}}$ mice expressing 15-PGDH (Myung et al. , 2006, Yan et al. , 2009). Our results in a chemical model of liver cancer induced with DEN and in the c-myc/TGF- $\alpha$ double transgenic mice show an important 15PGDH expression in the normal liver tissue but a decrease in the tumoral liver tissue (Llorente Izquierdo, Mayoral, 2011, Santoni-Rugiu, Jensen, 1998). In agreement with this data we observed a decreased viability in HepG2 cells expressing of 15-PGDH both in in vitro and in in vivo assays when explanted in nude mice, these effects being reverted after reduction of 15-PGDH levels.

Moreover, 15-PGDH expression has been also analyzed in human colorectal cancer and in human lung cancer. The expression pattern of 15-PGDH was reduced in microdissected early hyperplasic lesions and in colorectal carcinomas suggesting that 15-PGDH plays a role in prevention of tumor progression (Ding, Tong, 2005, Lou, Jing, 2012, Mann, Backlund, 2006). Our data in human biopsies of HCC show a high 15-PGDH expression in normal liver tissue and a lesser content in $c a .90 \%$, in tumor tissue biopsies from patients with HCC at the time that hepatoma cells overexpressing 15-PGDH show enhanced levels of pro-apoptotic proteins and to be more prone to activate caspase 3 . 
All this data suggest that 15-PGDH is a promising target for chemoprevention. Discovery and development of agents improving 15-PGDH expression and/or activity in HCC cells will provide additional approaches to cancer chemoprevention. Dexamethasone and other glucocorticoids were found to induce the expression of 15-PGDH in lung cancer cells (Tong and Tai, 2005). Non-steroidal anti-inflammatory drugs (NSAIDs) have been also evaluated exhibiting flurbiprofen the best capacity to induce 15-PGDH (Tai, Chi, 2011). Furthermore, peroxisome proliferator-activated receptor $\gamma$ ligands such as thiazolidinediones inhibit lung cancer cell growth by decreasing $\mathrm{PGE}_{2}$ production through induction of 15-PGDH (Hazra et al. , 2007). Rosiglitazone specifically decreased expression of Snail which is involved in 15PGDH suppression (Choudhary et al. , 2010). In this sense and while we were preparing this manuscript, Lu et al. reported that 15-PGDH inhibits HCC growth through an alternative mechanism involving 15-keto-PGE $2 / \mathrm{PPAR} \gamma$-mediated activation of $\mathrm{p} 21^{\mathrm{WAF} 1 / \mathrm{Cip} 1}$ signaling cascade (Lu et al. , 2013). Finally a promising target is the prostaglandin transporter (PTG) which may collaborate with 15-PGDH to inactivate $\mathrm{PGE}_{2}$ (Holla et al. , 2008).

In summary, we have found that the prostaglandin catabolic enzyme, 15-PGDH is downregulated in those human hepatoma cells with a high COX-2 and mPGES-1 expression, in chemical and genetic murine models of HCC and in human liver HCC biopsies and that over expression of 15-PGDH induces apoptosis in hepatoma cells. These results extend previous observations in other human cancers and suggest that 15-PGDH may function as a relevant tumor suppressor in $\mathrm{HCC}$. 


\section{Acknowledgements}

L C-S is supported by a Post-Doctoral fellowship from CONACYT, Mexico. NA is supported by Red Temática de Investigación Cooperativa en Enfermedades Cardiovasculares RECAVA. Instituto de Salud Carlos III (RD12/0042/0019). This work was supported by Ministry of Science and Innovation (SAF2010-16037, SAF2012-39732 and BFU2011-24760) and by Comunidad de Madrid (P2010/BMD-2378).

Conflict of Interest: None declared

\section{REFERENCES}

Backlund MG, Mann JR, Holla VR, Buchanan FG, Tai HH, Musiek ES, et al. 15Hydroxyprostaglandin dehydrogenase is down-regulated in colorectal cancer. J Biol Chem. 2005;280:3217-23.

Backlund MG, Mann JR, Holla VR, Shi Q, Daikoku T, Dey SK, et al. Repression of 15hydroxyprostaglandin dehydrogenase involves histone deacetylase 2 and snail in colorectal cancer. Cancer Res. 2008;68:9331-7.

Casado M, Callejas NA, Rodrigo J, Zhao X, Dey SK, Bosca L, et al. Contribution of cyclooxygenase 2 to liver regeneration after partial hepatectomy. FASEB J. 2001;15:2016-8. Cusimano A, Fodera D, Lampiasi N, Azzolina A, Notarbartolo M, Giannitrapani L, et al. Prostaglandin E2 receptors and COX enzymes in human hepatocellular carcinoma: role in the regulation of cell growth. Ann N Y Acad Sci. 2009;1155:300-8.

Cheng AS, Yu J, Lai PB, Chan HL, Sung JJ. COX-2 mediates hepatitis B virus X protein abrogation of p53-induced apoptosis. Biochem Biophys Res Commun. 2008;374:175-80. Choudhary R, Li H, Winn RA, Sorenson AL, Weiser-Evans MC, Nemenoff RA. Peroxisome proliferator-activated receptor-gamma inhibits transformed growth of non-small cell lung cancer cells through selective suppression of Snail. Neoplasia. 2010;12:224-34.

de La Coste A, Romagnolo B, Billuart P, Renard CA, Buendia MA, Soubrane O, et al. Somatic mutations of the beta-catenin gene are frequent in mouse and human hepatocellular carcinomas. Proc Natl Acad Sci U S A. 1998;95:8847-51.

Diaz-Munoz MD, Osma-Garcia IC, Fresno M, Iniguez MA. Involvement of PGE2 and the cAMP signalling pathway in the up-regulation of COX-2 and mPGES-1 expression in LPSactivated macrophages. The Biochemical journal. 2012;443:451-61.

Ding Y, Tong M, Liu S, Moscow JA, Tai HH. NAD+-linked 15-hydroxyprostaglandin dehydrogenase (15-PGDH) behaves as a tumor suppressor in lung cancer. Carcinogenesis. 2005;26:65-72.

Donnini S, Finetti F, Terzuoli E, Giachetti A, Iniguez MA, Hanaka H, et al. EGFR signaling upregulates expression of microsomal prostaglandin E synthase-1 in cancer cells leading to enhanced tumorigenicity. Oncogene. 2011.

Hazra S, Batra RK, Tai HH, Sharma S, Cui X, Dubinett SM. Pioglitazone and rosiglitazone decrease prostaglandin E2 in non-small-cell lung cancer cells by up-regulating 15hydroxyprostaglandin dehydrogenase. Mol Pharmacol. 2007;71:1715-20.

Holla VR, Backlund MG, Yang P, Newman RA, DuBois RN. Regulation of prostaglandin transporters in colorectal neoplasia. Cancer Prev Res (Phila). 2008;1:93-9.

Hoshida Y, Nijman SM, Kobayashi M, Chan JA, Brunet JP, Chiang DY, et al. Integrative transcriptome analysis reveals common molecular subclasses of human hepatocellular carcinoma. Cancer Res. 2009;69:7385-92. 
Kern MA, Haugg AM, Koch AF, Schilling T, Breuhahn K, Walczak H, et al. Cyclooxygenase- 2 inhibition induces apoptosis signaling via death receptors and mitochondria in hepatocellular carcinoma. Cancer Res. 2006;66:7059-66.

Kern MA, Schoneweiss MM, Sahi D, Bahlo M, Haugg AM, Kasper HU, et al. Cyclooxygenase-2 inhibitors suppress the growth of human hepatocellular carcinoma implants in nude mice. Carcinogenesis. 2004;25:1193-9.

Kern MA, Schubert D, Sahi D, Schoneweiss MM, Moll I, Haugg AM, et al. Proapoptotic and antiproliferative potential of selective cyclooxygenase-2 inhibitors in human liver tumor cells. Hepatology. 2002;36:885-94.

Koga H, Sakisaka S, Ohishi M, Kawaguchi T, Taniguchi E, Sasatomi K, et al. Expression of cyclooxygenase-2 in human hepatocellular carcinoma: relevance to tumor dedifferentiation. Hepatology. 1999;29:688-96.

Lee JS, Chu IS, Mikaelyan A, Calvisi DF, Heo J, Reddy JK, et al. Application of comparative functional genomics to identify best-fit mouse models to study human cancer. Nat Genet. 2004;36:1306-11.

Lim K, Han C, Dai Y, Shen M, Wu T. Omega-3 polyunsaturated fatty acids inhibit hepatocellular carcinoma cell growth through blocking beta-catenin and cyclooxygenase- 2 . Mol Cancer Ther. 2009;8:3046-55.

Lou LH, Jing DD, Lai YX, Lu YY, Li JK, Wu K. 15-PGDH is reduced and induces apoptosis and cell cycle arrest in gastric carcinoma. World J Gastroenterol. 2012;18:1028-37.

Lu D, Han C, Wu T. 15-PGDH inhibits hepatocellular carcinoma growth through 15-ketoPGE/PPARgamma-mediated activation of p21. Oncogene. 2013.

Llorente Izquierdo C, Mayoral R, Flores JM, Garcia-Palencia P, Cucarella C, Bosca L, et al. Transgenic mice expressing cyclooxygenase- 2 in hepatocytes reveal a minor contribution of this enzyme to chemical hepatocarcinogenesis. Am J Pathol. 2011;178:1361-73.

Llovet JM, Bruix J. Molecular targeted therapies in hepatocellular carcinoma. Hepatology. 2008;48:1312-27.

Mann JR, Backlund MG, Buchanan FG, Daikoku T, Holla VR, Rosenberg DW, et al. Repression of prostaglandin dehydrogenase by epidermal growth factor and snail increases prostaglandin E2 and promotes cancer progression. Cancer Res. 2006;66:6649-56. Martin-Sanz P, Callejas NA, Casado M, Diaz-Guerra MJ, Bosca L. Expression of cyclooxygenase-2 in foetal rat hepatocytes stimulated with lipopolysaccharide and proinflammatory cytokines. Br J Pharmacol. 1998;125:1313-9.

Mayoral R, Fernandez-Martinez A, Bosca L, Martin-Sanz P. Prostaglandin E2 promotes migration and adhesion in hepatocellular carcinoma cells. Carcinogenesis. 2005;26:753-61. Moore AE, Greenhough A, Roberts HR, Hicks DJ, Patsos HA, Williams AC, et al. HGF/Met signalling promotes PGE(2) biogenesis via regulation of COX-2 and 15-PGDH expression in colorectal cancer cells. Carcinogenesis. 2009;30:1796-804.

Myung SJ, Rerko RM, Yan M, Platzer P, Guda K, Dotson A, et al. 15-Hydroxyprostaglandin dehydrogenase is an in vivo suppressor of colon tumorigenesis. Proc Natl Acad Sci U S A. 2006;103:12098-102.

Na HK, Park JM, Lee HG, Lee HN, Myung SJ, Surh YJ. 15-Hydroxyprostaglandin dehydrogenase as a novel molecular target for cancer chemoprevention and therapy. Biochem Pharmacol. 2011;82:1352-60.

Nunez O, Fernandez-Martinez A, Majano PL, Apolinario A, Gomez-Gonzalo M, Benedicto I, et al. Increased intrahepatic cyclooxygenase 2, matrix metalloproteinase 2, and matrix metalloproteinase 9 expression is associated with progressive liver disease in chronic hepatitis C virus infection: role of viral core and NS5A proteins. Gut. 2004;53:1665-72.

Rudnick DA, Perlmutter DH, Muglia LJ. Prostaglandins are required for CREB activation and cellular proliferation during liver regeneration. Proc Natl Acad Sci U S A. 2001;98:8885-90. 
Santoni-Rugiu E, Jensen MR, Thorgeirsson SS. Disruption of the $\mathrm{pRb} / \mathrm{E} 2 \mathrm{~F}$ pathway and inhibition of apoptosis are major oncogenic events in liver constitutively expressing c-myc and transforming growth factor alpha. Cancer Res. 1998;58:123-34.

Sasaki Y, Kamei D, Ishikawa Y, Ishii T, Uematsu S, Akira S, et al. Microsomal prostaglandin E synthase-1 is involved in multiple steps of colon carcinogenesis. Oncogene. 2011;31:294352.

Simmons DL, Botting RM, Hla T. Cyclooxygenase isozymes: the biology of prostaglandin synthesis and inhibition. Pharmacol Rev. 2004;56:387-437.

Song HJ, Myung SJ, Kim IW, Jeong JY, Park YS, Lee SM, et al. 15-hydroxyprostaglandin dehydrogenase is downregulated and exhibits tumor suppressor activity in gastric cancer. Cancer Invest. 2011;29:257-65.

Subbaramaiah K, Yoshimatsu K, Scherl E, Das KM, Glazier KD, Golijanin D, et al. Microsomal prostaglandin E synthase-1 is overexpressed in inflammatory bowel disease. Evidence for involvement of the transcription factor Egr-1. J Biol Chem. 2004;279:12647-58. Tai HH. Prostaglandin catabolic enzymes as tumor suppressors. Cancer Metastasis Rev. 2011;30:409-17.

Tai HH, Chi X, Tong M. Regulation of 15-hydroxyprostaglandin dehydrogenase (15-PGDH) by non-steroidal anti-inflammatory drugs (NSAIDs). Prostaglandins Other Lipid Mediat. 2011;96:37-40.

Tai HH, Cho H, Tong M, Ding Y. NAD+-linked 15-hydroxyprostaglandin dehydrogenase: structure and biological functions. Curr Pharm Des. 2006;12:955-62.

Tong M, Ding Y, Tai HH. Histone deacetylase inhibitors and transforming growth factor-beta induce 15-hydroxyprostaglandin dehydrogenase expression in human lung adenocarcinoma cells. Biochem Pharmacol. 2006;72:701-9.

Tong M, Tai HH. Synergistic induction of the nicotinamide adenine dinucleotide-linked 15hydroxyprostaglandin dehydrogenase by an androgen and interleukin-6 or forskolin in human prostate cancer cells. Endocrinology. 2004;145:2141-7.

Tong M, Tai HH. 15-Hydroxyprostaglandin dehydrogenase can be induced by dexamethasone and other glucocorticoids at the therapeutic level in A549 human lung adenocarcinoma cells. Arch Biochem Biophys. 2005;435:50-5.

Wolf I, O'Kelly J, Rubinek T, Tong M, Nguyen A, Lin BT, et al. 15-hydroxyprostaglandin dehydrogenase is a tumor suppressor of human breast cancer. Cancer Res. 2006;66:7818-23.

Yamamoto H, Kondo M, Nakamori S, Nagano H, Wakasa K, Sugita Y, et al. JTE-522, a cyclooxygenase-2 inhibitor, is an effective chemopreventive agent against rat experimental liver fibrosis1. Gastroenterology. 2003;125:556-71.

Yan M, Myung SJ, Fink SP, Lawrence E, Lutterbaugh J, Yang P, et al. 15-

Hydroxyprostaglandin dehydrogenase inactivation as a mechanism of resistance to celecoxib chemoprevention of colon tumors. Proc Natl Acad Sci U S A. 2009;106:9409-13.

Yan M, Rerko RM, Platzer P, Dawson D, Willis J, Tong M, et al. 15-Hydroxyprostaglandin dehydrogenase, a COX-2 oncogene antagonist, is a TGF-beta-induced suppressor of human gastrointestinal cancers. Proc Natl Acad Sci U S A. 2004;101:17468-73.

Yang L, Amann JM, Kikuchi T, Porta R, Guix M, Gonzalez A, et al. Inhibition of epidermal growth factor receptor signaling elevates 15-hydroxyprostaglandin dehydrogenase in nonsmall-cell lung cancer. Cancer Res. 2007;67:5587-93. 


\section{FIGURE LEGENDS}

Fig. 1. 15-PGDH and COX-2 correlate inversely in HCC cell lines. Cells were plated in 100mm dishes and grown to $60 \%$ confluence in culture medium supplemented with $10 \%$ FBS and subsequently placed in serum starved for $6 \mathrm{~h}$. Total cellular extracts or RNA were obtained from hepatic cells lines. (A) COX-2 (72kDa), mPGES-1 (17 kDa) and 15-PGDH (25 kDa) protein expression were analyzed by Western blot of cell lysates $(25 \mu \mathrm{g} / \mathrm{lane})$. The protein expression was normalized to that $\beta$-actin. Densitometric analysis of COX-2, mPGES-1 and 15-PGDH expression are referring to human hepatocytes $(\mathrm{HH})$ as 1 and expressed as fold basal expression. A representative Western blot is shown. (B-C-D) COX-2, mPGES-1 and 15-PGDH mRNA were analyzed by real-time PCR. The mRNA amounts were calculated as relative expression (RE) and normalized with 36b4 mRNA levels. Values represent relative fold change to HH. (E) Prostaglandin $\mathrm{E}_{2}$ concentration was determined by enzyme immunoassay using the cell supernatants. (F) The inverse correlation between COX-2/15PGDH protein expression ratio in hepatic cells lines is graphically depicted. The coefficient of determination $\left(\mathrm{R}^{2}\right)$ was calculated. Data are means $\pm \mathrm{SD}$ of at least three independent experiments. $* * \mathrm{P} \leq 0.01, * \mathrm{P} \leq 0.05$ vs. $\mathrm{HH}$.

\section{Fig. 2. EGF and HGF promote an increase of COX-2 and MPGES-1 and decrease 15-} PGDH protein levels in the CHL hepatocyte-like cell line. Cell cultures to $60 \%$ confluence were placed in serum starved for $6 \mathrm{~h}$. Subsequently, the culture medium was replaced with fresh medium containing 1\% FBS and then CHL cells were treated with different concentrations of EGF or HGF for $48 \mathrm{~h}$ or with $50 \mathrm{ng} / \mathrm{ml}$ of EGF at different time points and then obtained cells lysates or RNA. COX-2, mPGES-1 and 15-PGDH protein expression were determined as described in Fig. 1. (A) Analysis COX-2, mPGES-1 and 15-PGDH expression 
by Western blot after treatment with different concentrations of EGF (B) Analysis COX-2, mPGES-1 and 15-PGDH expression by Western blot after $50 \mathrm{ng} / \mathrm{ml}$ of EGF at different time points (C) Analysis of COX-2 and mPGES-1 mRNA after $6 \mathrm{~h}$ of EGF treatment and 15PGDH mRNA at different time points by real-time PCR. The mRNA amounts were calculated as relative expression and normalized with $36 \mathrm{~b} 4 \mathrm{mRNA}$ levels. Values represent relative fold change to unstimulated cells. (D) Analysis COX-2, mPGES-1 and 15-PGDH expression by Western blot after treatment with different concentrations of HGF. Data are means $\pm \mathrm{SD}$ of at least three independent experiments. $* * \mathrm{P} \leq 0.01, * \mathrm{P} \leq 0.05$ vs. unstimulated cells.

Fig. 3. EGF downregulates 15-PGDH through EGFR, PI3K, MEK /ERK-MAPK and p38MAPK signaling pathways in CHL cells. CHL cells were treated or not with different pharmacological inhibitors during $1 \mathrm{~h}$ as described in methods and subsequently stimulated with $50 \mathrm{ng} / \mathrm{ml}$ of EGF for $48 \mathrm{~h}$. COX-2 and 15-PGDH protein expression were analyzed as described in Fig. 1 and 2 (A) Analysis of COX-2 and 15-PGDH expression by Western blot (B) effect of $5 \mu \mathrm{M}$ DFU and $5 \mu \mathrm{M} \mathrm{PGE}_{2}$ on the 15 -PGDH expression. Data are means $\pm \mathrm{SD}$ of at least three independent experiments. ${ }^{*} * \mathrm{P} \leq 0.01, * \mathrm{P} \leq 0.05$ vs. unstimulated cells. $\# \mathrm{P} \leq 0.05$ vs. stimulated cells with EGF.

Fig. 4. 15-PGDH expression decreases HCC cell viability and induces apoptosis. HepG2 cells were seeded in a 6 -well plate $\left(3 \times 10^{5}\right.$ cells/well) at $60 \%$ confluence and transfected with pcDNA3/15-PGDH ( $3 \mu \mathrm{g})$, pcDNA3 $(3 \mu \mathrm{g}), 30 \mathrm{nM}$ siPGDH or $30 \mathrm{nM}$ scRNA as described in Methods (A) Analysis of 15-PGDH expression by for Western blot. (B) Cell viability (fold basal) was determined by the MTT assay. (C-D) Analysis of cell-cycle distribution by PI staining of HepG2 transfected cells. The analysis was performed using the percent of frequencies in each cycle phase (number of cells vs. relative intensity). (E) Apoptosis was 
detected by flow cytometry using annexin V-FITC apoptosis detection kit. (F) Caspase-3 activity was determined by CaspGLOW fluorescein active caspase- 3 staining kit. The protease activity is represented by fluorescence arbitrary units (a.u.)/ $\mu \mathrm{g}$ of total protein, 4.76 a.u. corresponding to Lipofectamine is considered as 1. (G-H) Analysis of pro-apoptotic and anti-apoptotic proteins expression by Western blot. Data are means \pm SD of at least three independent experiments. ${ }^{*} \mathrm{P} \leq 0.01,{ }^{*} \mathrm{P} \leq 0.05$ vs. lipofectamine. HepG2 transfected cells with scRNA and siPGDH (I) or pcDNA3 and pcDNA3/15-PGDH (J) were subcutaneously injected into athymic $n u / n u$ mice. The mice were followed by the observation of xenograft growth for 3 weeks. Tumor growth was measured every 2-3 days. Tumor volume (V) was monitored by measuring the length (L) and width (W) with calipers and calculated with the formula $\left(\mathrm{Lx} \mathrm{W}^{2}\right) \times$ 0.5. At 21 days after injection, mice were killed and tumors were weighed after necropsy. $* \mathrm{P} \leq 0.05 v s$. the scRNA or $v s$. the pcDNA3. Data are means \pm SD of eight animals per condition.

Fig. 5. 15-PGDH is decreased in murine models of HCC. To induce HCC, DEN (25 mg/kg) was injected i.p. into 14 day-old mice. Mice were sacrificed at 10 or 40 weeks after DEN treatment and then the liver obtained for protein extracts. (A) Morphometric analysis of tumor foci number and maximal size at 10 and 40 wk of DEN treatment. (B) H\&E analysis of liver sections in mice at 10 and 40 wk of DEN treatment. Preneoplastic lesion (left panel) and HCC lesions (right panel) are indicated by arrows. Inset: Lung metastases (C) Western blot representative of 15-PGDH expression in tumor (T) or non-tumor (NT) liver tissue after DEN treatment compared with normal liver and normalized with $\alpha$ p85. (D) Morphometric analysis of tumor foci number and maximal size at 5 and 9 months-old-age c-myc/TGF- $\alpha$ mice. (E) H\&E staining of liver sections showing $\mathrm{HCC}$ with trabecular pattern, cytological atypias and nuclear polymorphisms in c-myc/TGF- $\alpha$ mice at 9 months-old-age (right panel). A sharply demarcated hepatocellular adenoma with enlarged eosinophilic hepatocytes is shown at 5 
months-old-age (left panel) (F) 15-PGDH protein expression in liver homogenates from cmyc/TGF- $\alpha$ mice at 5 and 9 months-old-age detected by Western blot and normalized with $\alpha p 85$. The double transgenic was verified by c-myc and TGF- $\alpha$ protein expression. The photographs were taken with a Color View Camera in a Vanox Olympus microscope at 20X magnification. The bar corresponds to $100 \mu \mathrm{m}$ and lesions are indicated by arrows. Data are means \pm SD of the number of animals indicated in supplementary Table I and II per condition. $* * \mathrm{P} \leq 0.01, * \mathrm{P} \leq 0.05 v s$. the corresponding matched animals.

\section{Fig. 6. 15-PGDH mRNA and protein expression is down-regulated in HCC human}

biopsies. 15-PGDH expression was analyzed in 11 clinical samples of human hepatocellular carcinomas and normal liver in a tissue microarray for immunohistochemistry analysis. (A) Representative microphotographs of normal liver (a) and HCC samples (b-c).

Microphotographs were taken with an EnVision FLEX system at 20X magnification. The bar corresponds to $100 \mu \mathrm{m}$ (B) The immunoreactivity of 15-PGDH expression was quantified as described in material and methods. The graphs represent the fold basal of immunostained of HCC samples (b-1) compared with normal liver (a). (C) The immunoreactivity measures were pooled and then compared with normal liver. The graphs represent the fold basal of immunostained of HCC samples compared with normal liver. Data are means \pm SD of 11 HCC samples. ** $\mathrm{P} \leq 0.01$ vs. normal liver tissue. (D) 15-PGDH mRNA expression was analyzed in 18 individual tumoral and paired non-tumoral HCC tumors using real-time PCR. $* \mathrm{P} \leq 0.05$ vs. NT samples. 
Figure 1

A

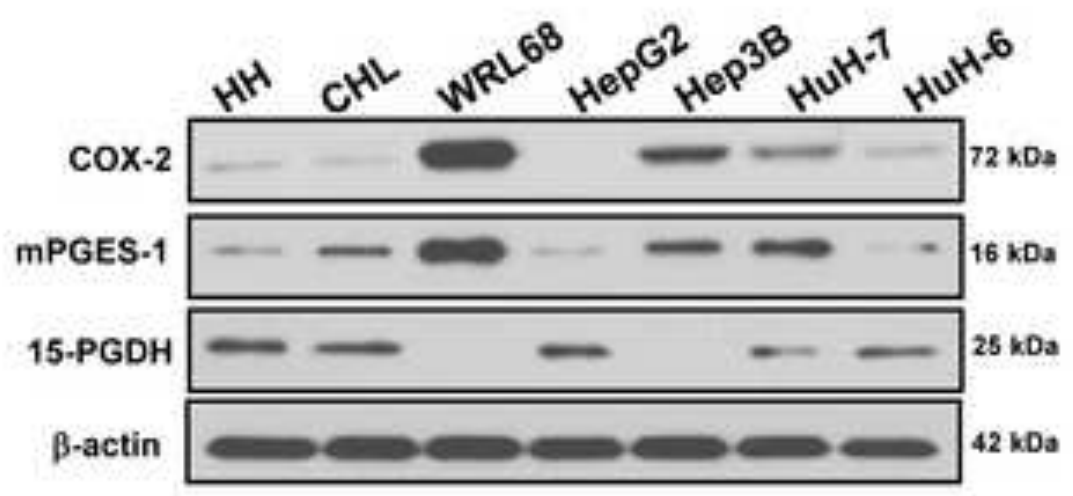

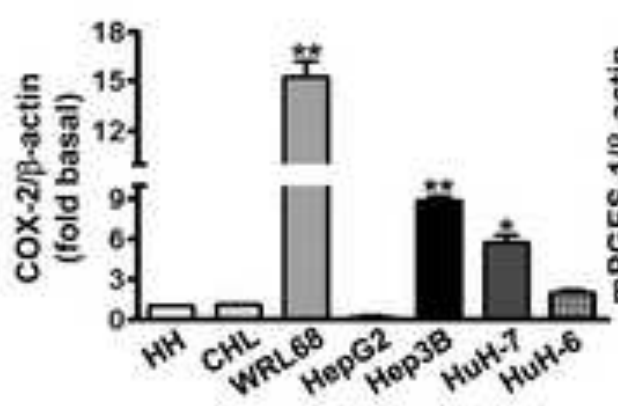

B

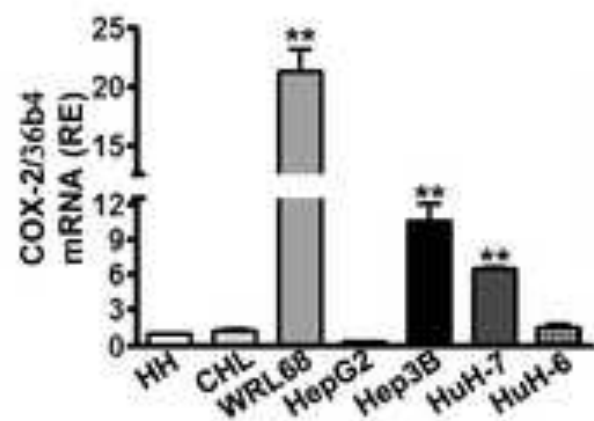

E

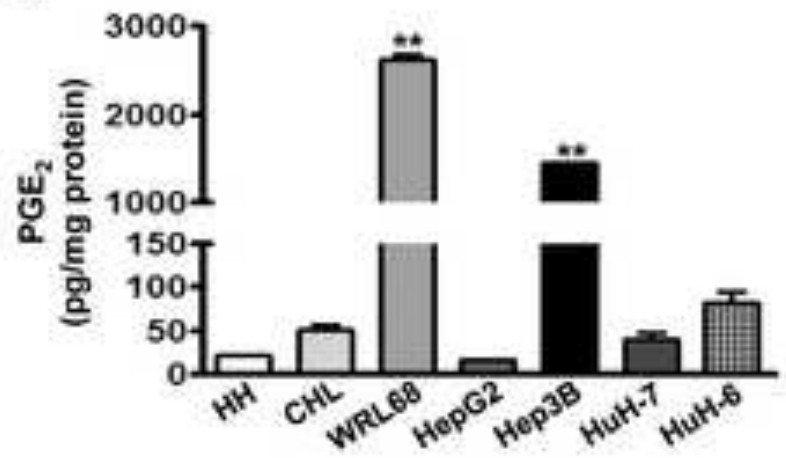

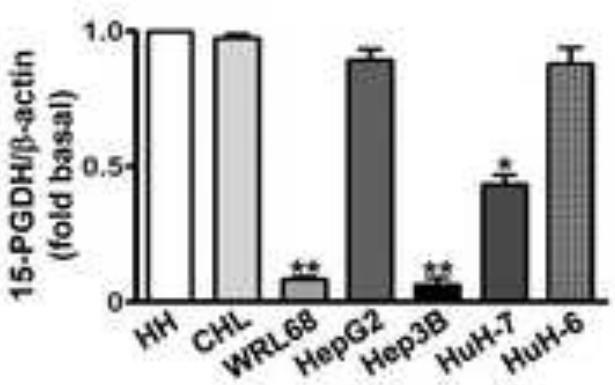

D
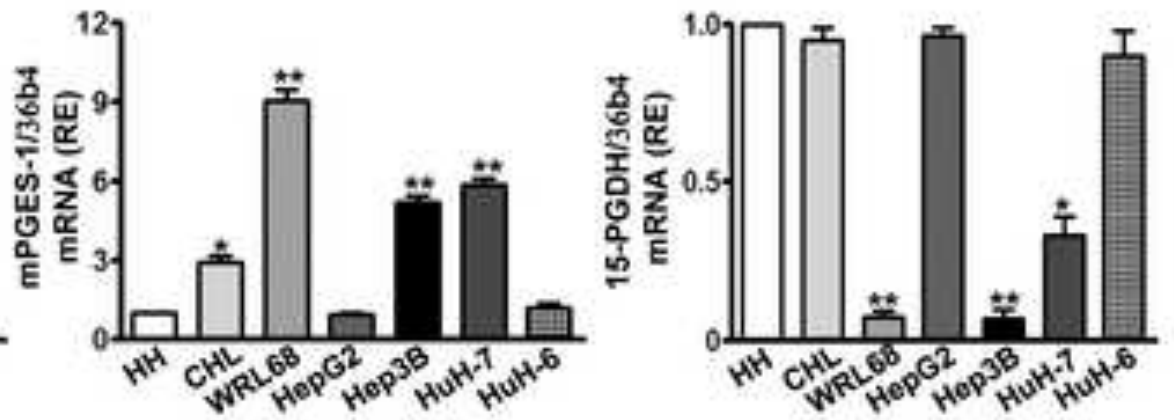

$\mathbf{F}$

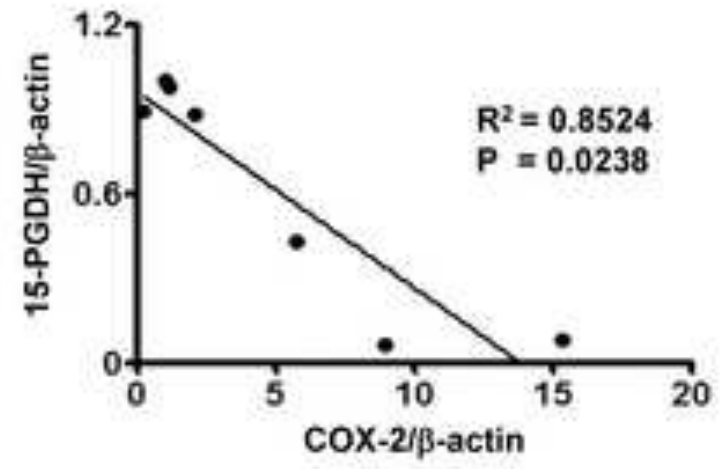


Figure 2

A

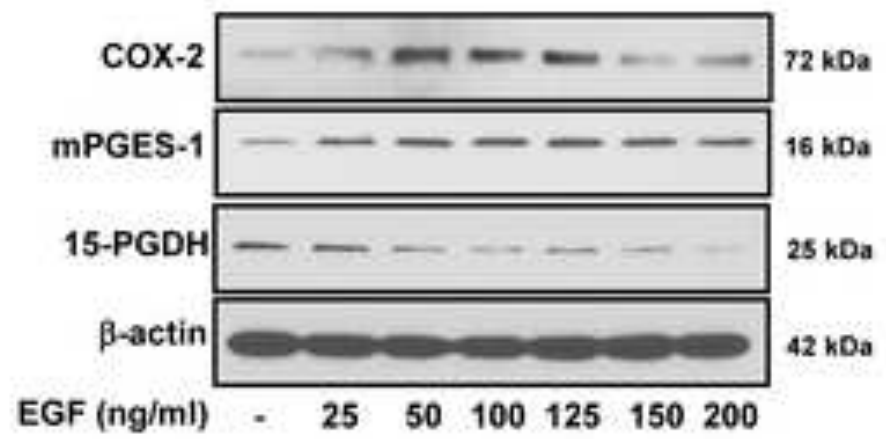

B

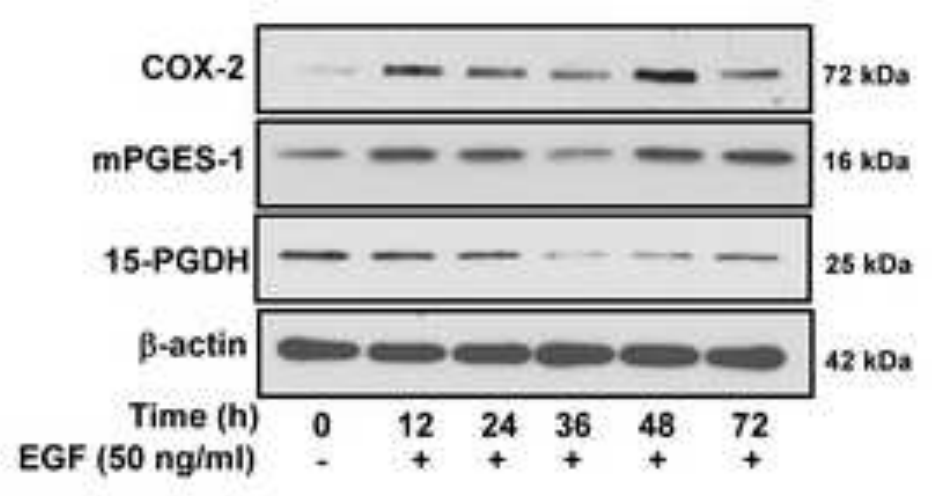

C

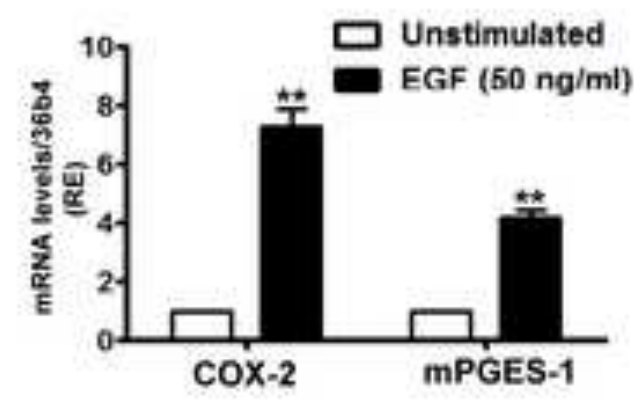

D

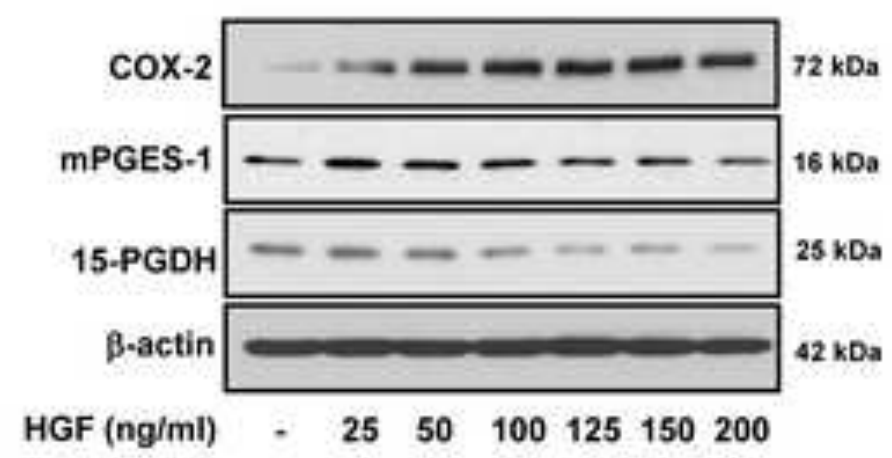

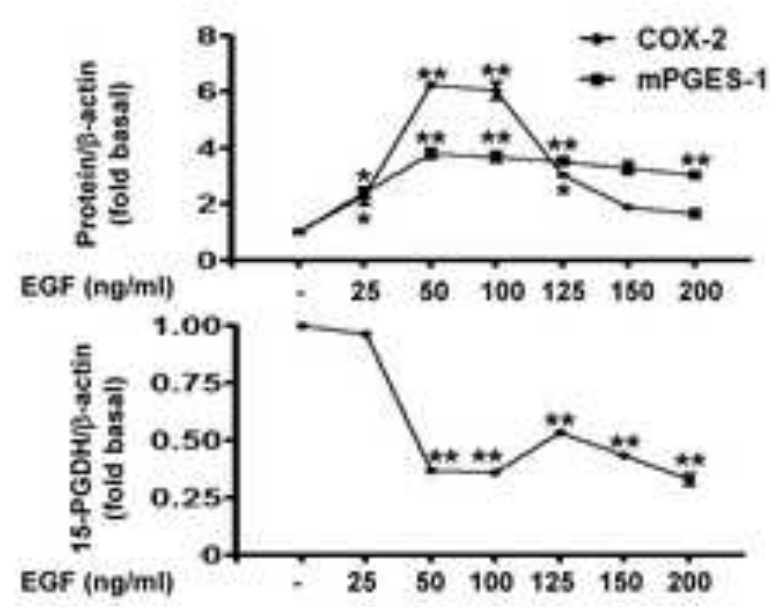
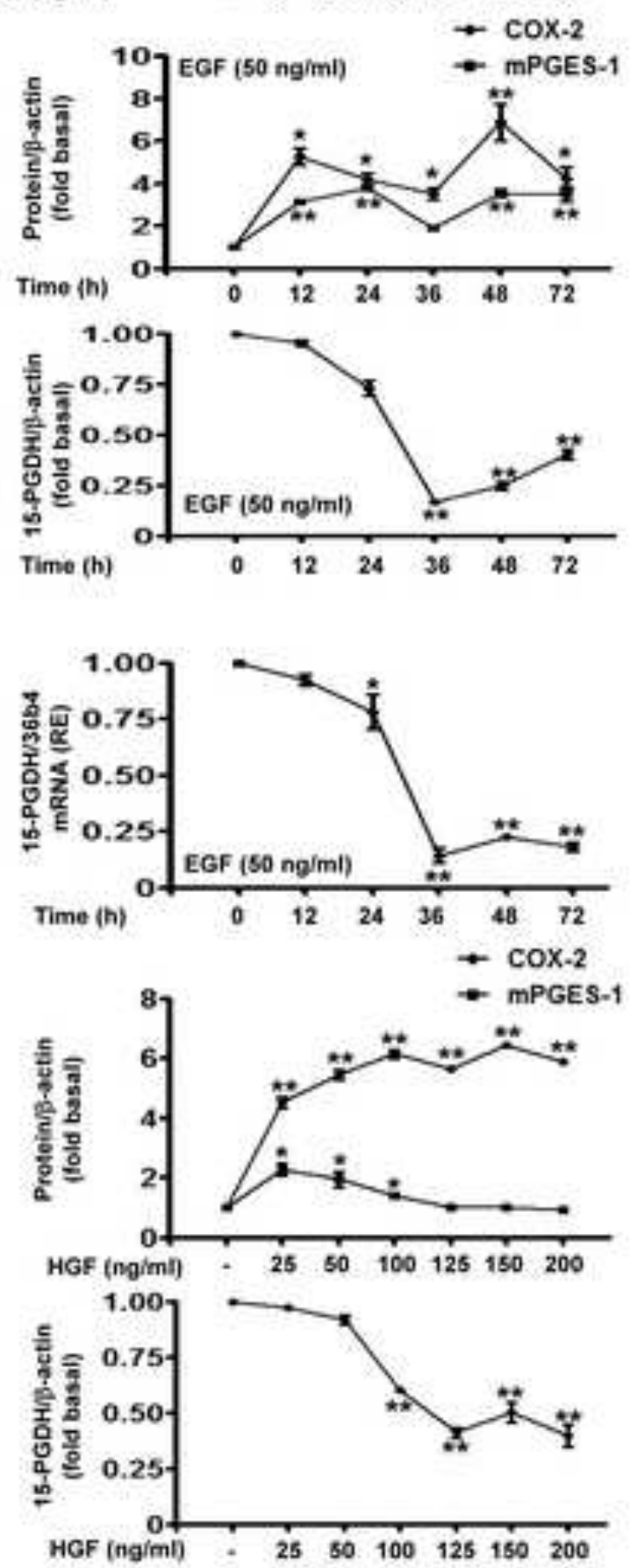
Figure 3

A
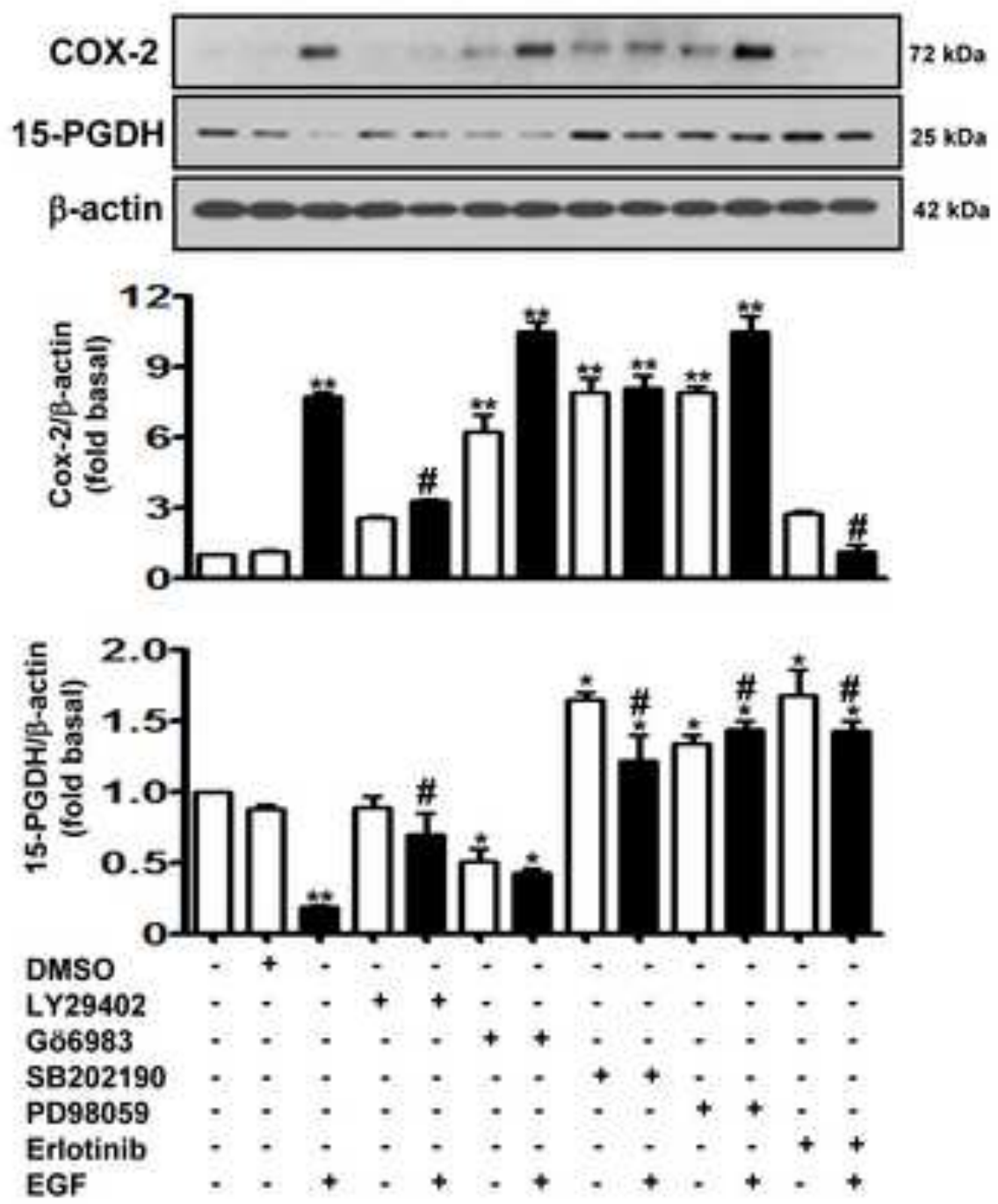

B
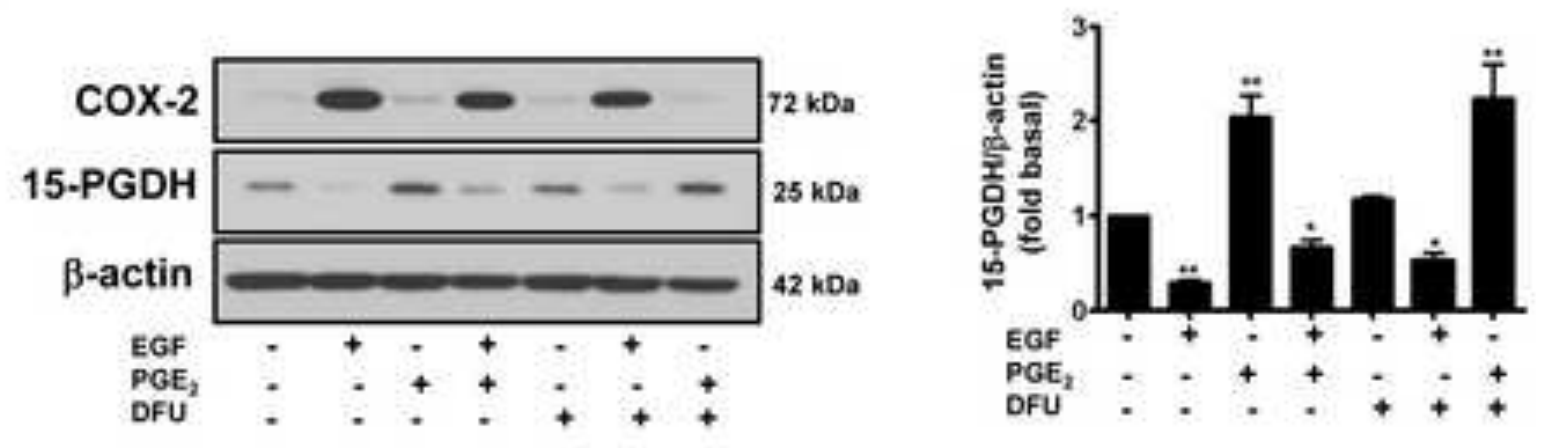


\section{Figure 4}

A

E
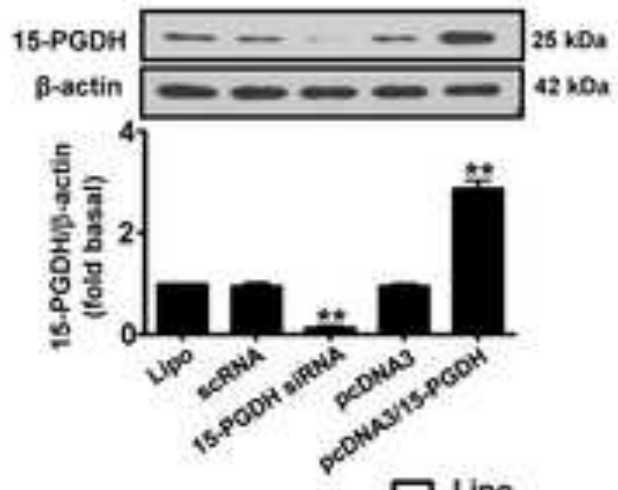

C
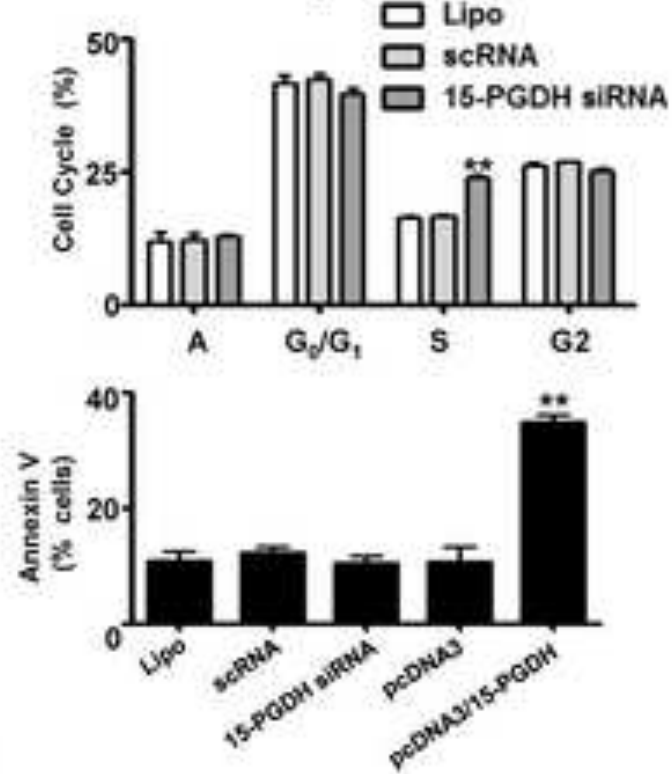

G
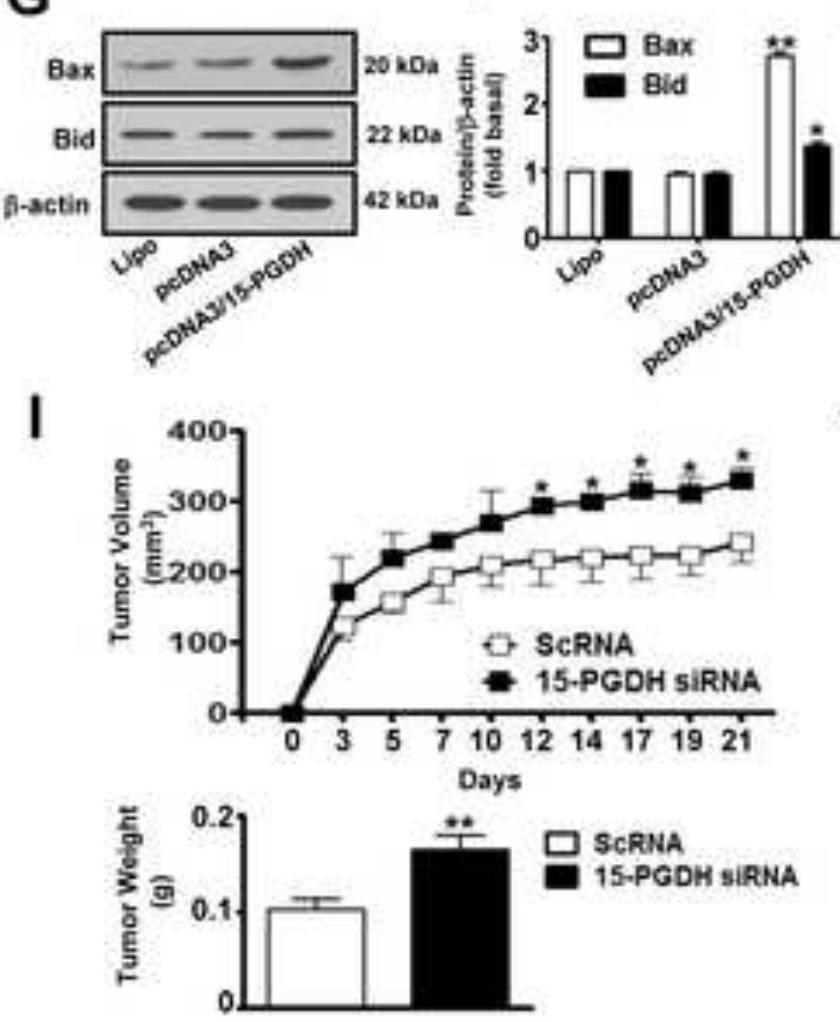

B

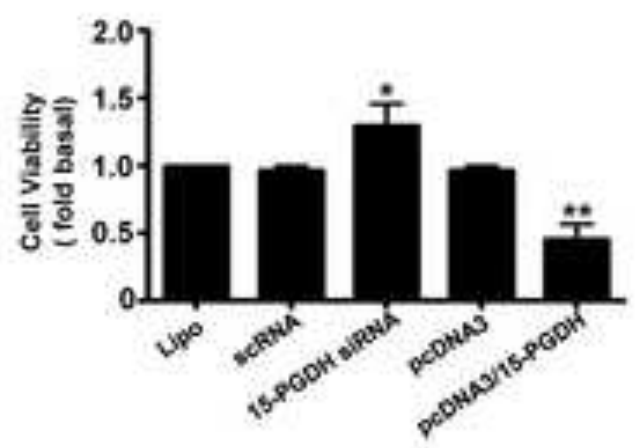

D

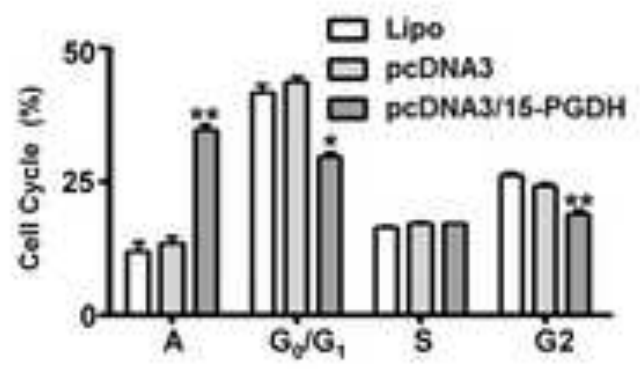

$\mathbf{F}$

$\mathrm{H}$
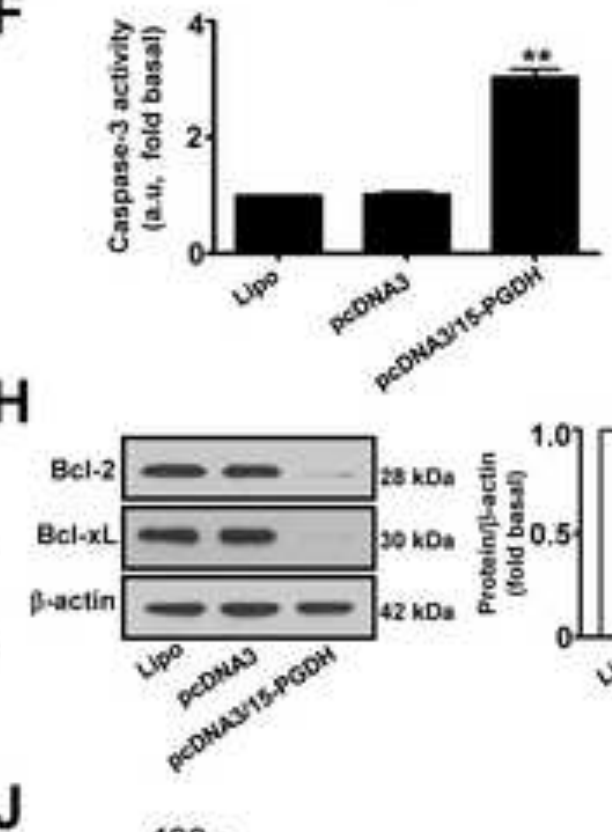

J
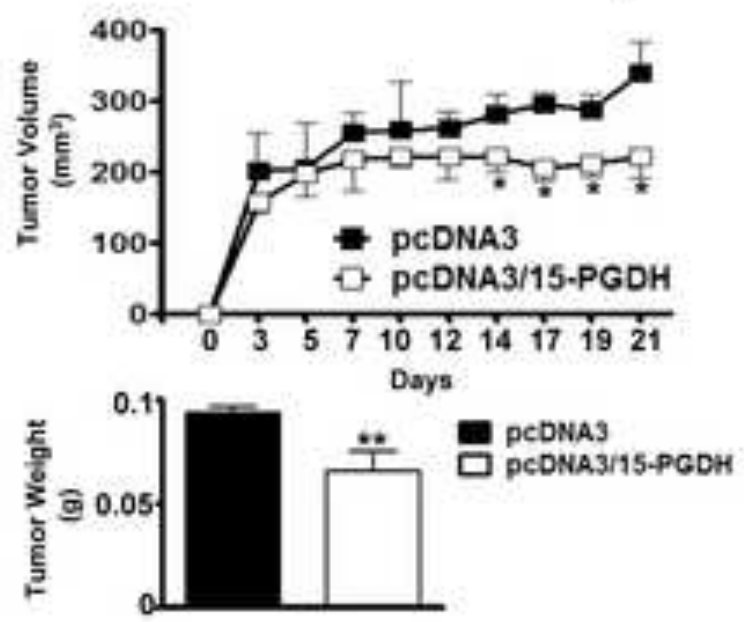
Figure 5

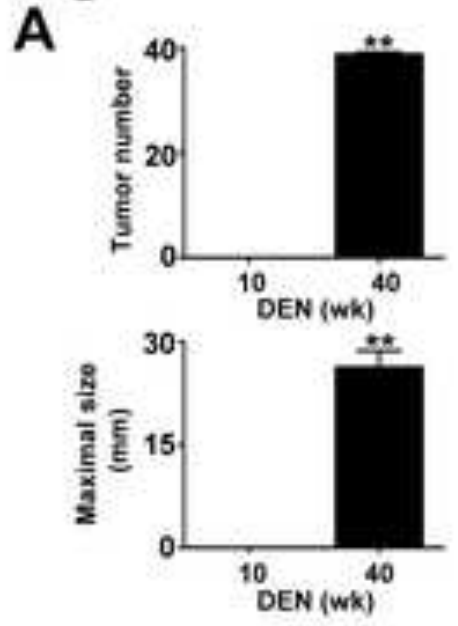

C

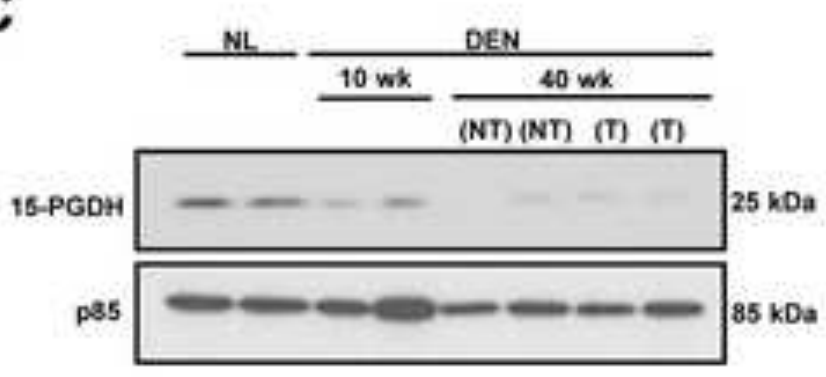

D
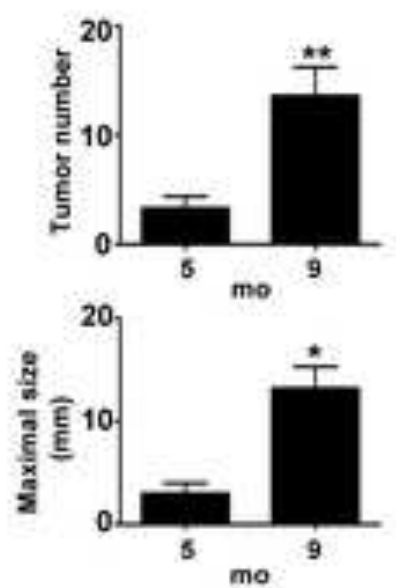

$\mathbf{F}$

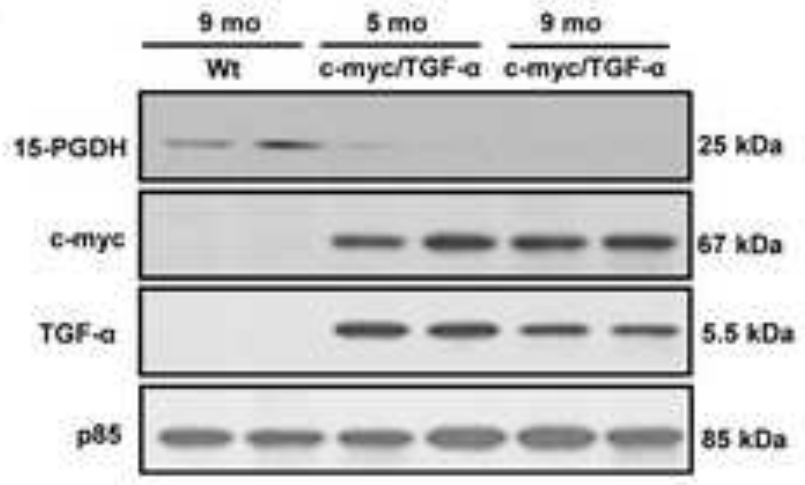

B

E
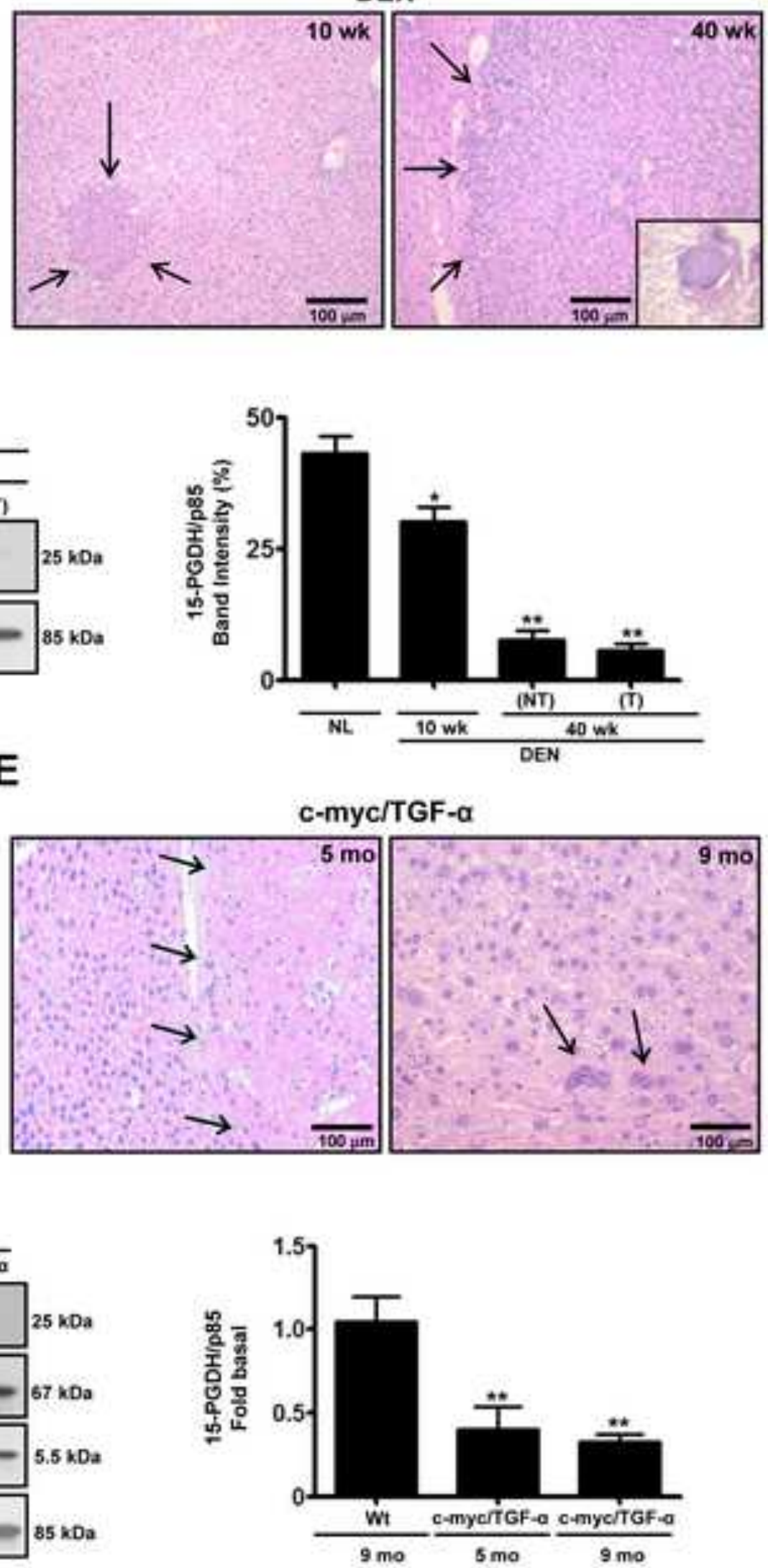
Figure(s)

Click here to download high resolution image

Figure 6
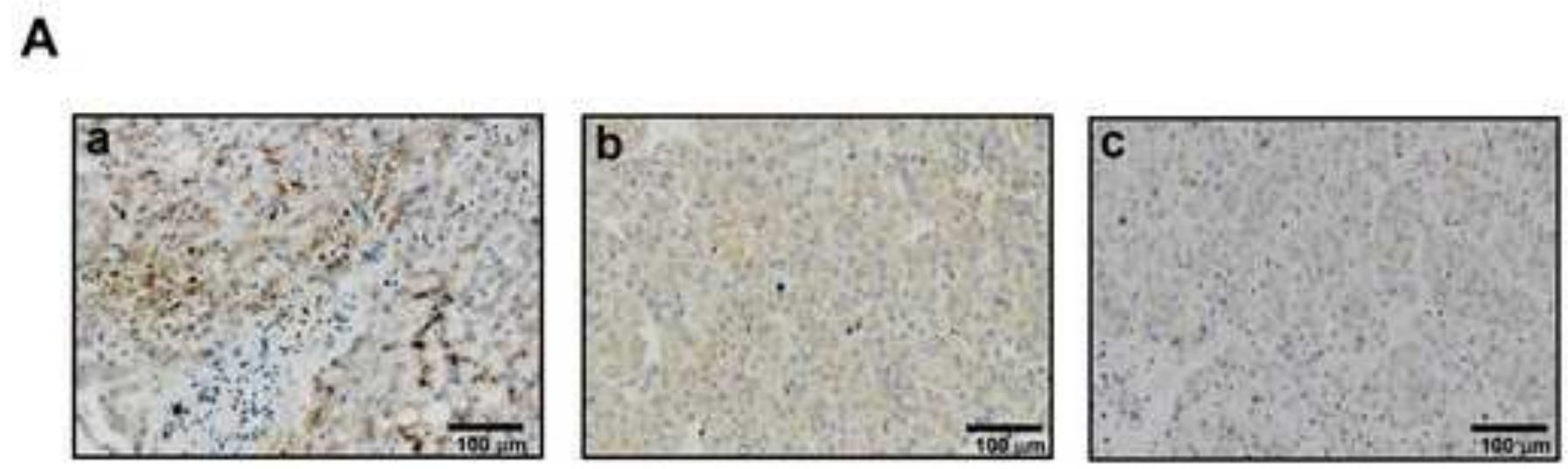

B

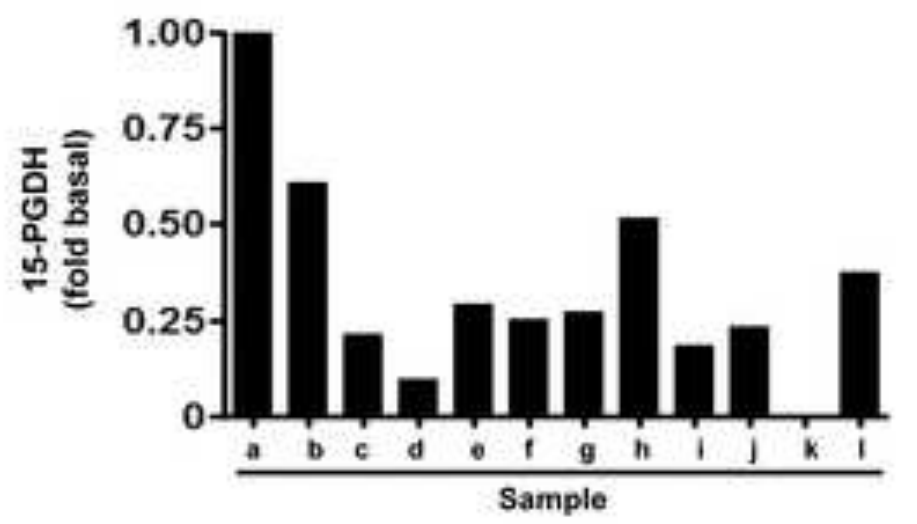

C

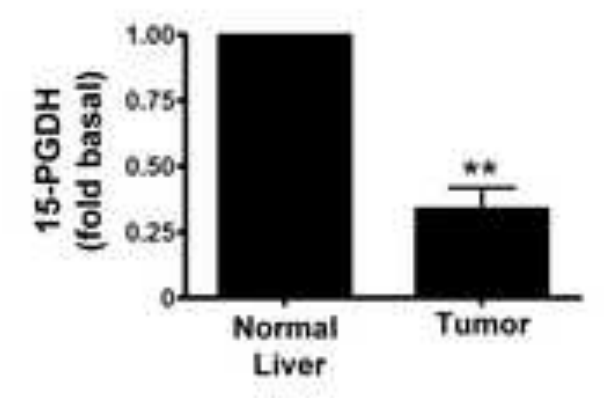

D

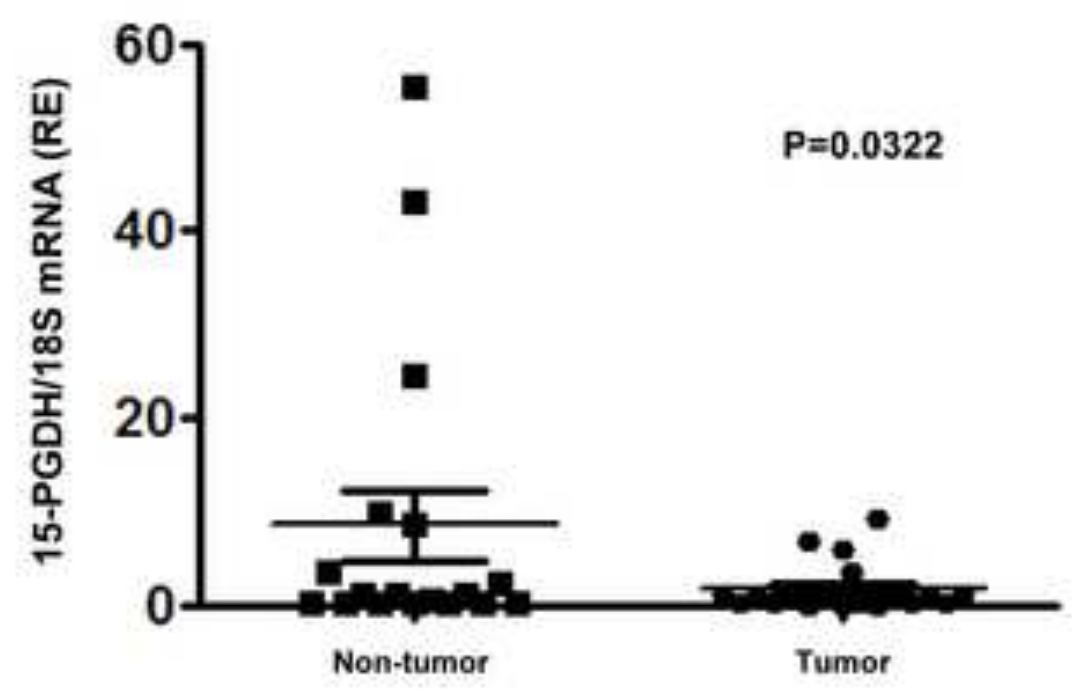

